\title{
A comparative study of structures and structural transitions of secondary transporters with the LeuT fold
}

\author{
Gunnar Jeschke
}

Received: 6 January 2012/Revised: 29 February 2012 / Accepted: 7 March 2012 / Published online: 3 May 2012

(C) The Author(s) 2012. This article is published with open access at Springerlink.com

\begin{abstract}
Secondary active transporters from several protein families share a core of two five-helix inverted repeats that has become known as the LeuT fold. The known highresolution protein structures with this fold were analyzed by structural superposition of the core transmembrane domains (TMDs). Three angle parameters derived from the mean TMD axes correlate with accessibility of the central binding site from the outside or inside. Structural transitions between distinct conformations were analyzed for four proteins in terms of changes in relative TMD arrangement and in internal conformation of TMDs. Collectively moving groups of TMDs were found to be correlated in the covariance matrix of elastic network models. The main features of the structural transitions can be reproduced with the $5 \%$ slowest normal modes of anisotropic elastic network models. These results support the rocking bundle model for the major conformational change between the outward- and inwardfacing states of the protein and point to an important role for the independently moving last TMDs of each repeat in occluding access to the central binding site. Occlusion is also supported by flexing of some individual TMDs in the collectively moving bundle and hash motifs.
\end{abstract}

Special Issue: Structure, function, folding and assembly of membrane proteins-Insight from Biophysics.

Electronic supplementary material The online version of this article (doi:10.1007/s00249-012-0802-z) contains supplementary material, which is available to authorized users.

G. Jeschke $(\square)$

Laboratory of Physical Chemistry, ETH Zürich,

Wolfgang-Pauli-Strasse 10, 8093 Zürich, Switzerland

e-mail: gunnar.jeschke@phys.chem.ethz.ch

URL: http://www.epr.ethz.ch/
Keywords Membrane proteins - Secondary active transport - Elastic network models · Protein structure . Protein dynamics

\section{Introduction}

Transport of substrates across biological membranes against a concentration gradient is required for cell metabolism and signaling between cells. In secondary active transport a membrane protein couples this energetically unfavorable substrate translocation to energetically favorable translocation of an ion or several ions along an electrochemical gradient. The substrate and ion(s) may move in the same direction (symport) or opposite direction (antiport). In both cases transport is thought to conform to the alternating access model (Jardetzky 1966). This model stipulates that a central substrate binding site in the protein is accessible either from the outside or from the inside of the membrane. Detailed understanding of the transport process then depends on characterization of the structural transitions involved in substrate and ion binding and, in particular, in the switch from the outward-facing to the inward-facing states (Klingenberg 2006).

For decades, such detailed understanding was hampered by a lack of high-resolution structures of any secondary active transporter. With advances in crystal structure determination this situation has changed (Abramson et al. 2003; Huang et al. 2003). This is particularly true for the LeuT fold, which was first observed for a bacterial amino acid transporter that is a homolog of eukaryotic neurotransmitters (Yamashita et al. 2005, Zhou et al. 2007; Singh et al. 2008). The main characteristic of the LeuT fold is the occurrence of two five-helix inverted repeats that include the substrate and ion translocation channel. Such a 
ten-helix core consisting of two inverted repeats was later found in sodium/substrate symporters (Faham et al. 2008; Weyand et al. 2008; Ressl et al. 2009), a cooperative substrate/product antiporter (Schulze et al. 2010; Tang et al. 2010), and proton/substrate antiporters (Fang et al. 2009; Shaffer et al. 2009; Gao et al. 2010; Kowalczyk et al. 2011) from different protein families, despite insignificant sequence homology between proteins that share this fold. Indeed, sequence homology is weak even between the two inverted repeats of a given protein.

Based on these crystal structures for the LeuT fold a number of modeling studies were performed (Forrest et al. 2008; Watanabe et al. 2010; Zhao et al. 2010; Shi and Weinstein 2010; Adelman et al. 2011, Koldsø et al. 2011), and the transport mechanism was discussed in general (Abramson and Wright 2009; Forrest et al. 2011, Rudnick 2011).

However, as the crystal structures are static snapshots of a dynamic process, they do not imply a unique mechanistic interpretation. Crystal structures do not exist for all conformations that are relevant during the transport process in any of the secondary transporters. Furthermore, crystallization often requires harsh detergents or physiologically unrealistic substrate concentrations. This may lead to stabilization of conformations that are off-path with respect to substrate and ion translocation (Quick et al. 2009; Cross et al. 2011; Mchaourab et al. 2011). Perhaps not surprisingly, this has led to a controversy about the main movement between the outward-facing and inward-facing conformations, with a rocker-switch rigid-body motion of a bundle of helices (Forrest et al. 2008) and systematic changes in the tilt angles of partially unwound helices (Yamashita et al. 2005; Zhao et al. 2010, 2011) being possible contenders. A recent simulation study on a homology model for the related human serotonin transporter indicates that both types of motions contribute (Koldsø et al. 2011).

In this situation, further experimental information on the structural changes is required. Such information can be obtained on secondary transporters in environments that are closer to biological membranes by probe techniques, such as fluorescence resonance energy transfer (Zhao et al. 2010) and electron paramagnetic resonance (EPR) spectroscopy (Jeschke et al. 2004; Hilger et al. 2005, 2007, 2009; Smirnova et al. 2007; Claxton et al. 2010). With such techniques structural changes can be observed on adding ions or substrate. Pulse EPR measurements of distance distributions (Jeschke and Polyhach 2007) can reveal that some functional states are broad ensembles of structures while others are better defined (Claxton et al. 2010; Mchaourab et al. 2011).

Conclusions from probe techniques are mainly limited by a small number of distance constraints. This lack of detailed information results from the fact that each constraint requires construction, labeling, and measurement of an individual mutant protein. Therefore, pairs of labeling sites need to be selected very carefully, which may require hypotheses on the expected structural transitions. Although discussions of the transport mechanism in the LeuT fold exist (Abramson and Wright 2009; Forrest et al. 2011; Rudnick 2011), a systematic comparison of all existing structural information is still missing. Such a study can provide sets of testable predictions on distance changes induced by substrate and ion addition.

Furthermore, modeling of a structural transition from sparse distance constraints requires an approach that reduces the number of degrees of freedom. Such state space reduction can be based on the concept of essential protein dynamics, which stipulates that functionally relevant largescale conformational changes are restricted to a few normal modes (Amadei et al. 1993). These modes are characterized by high collectivity of the motion and are associated with low vibration frequencies. Low-frequency normal modes can be predicted with reasonable precision and low computational effort from a single structure by coarsegrained elastic network models (ENM) (Bahar et al. 2010). For several pairs of soluble protein structures it has been demonstrated that structural transitions can be modeled reasonably well by driving a $\mathrm{C}^{\alpha}$ atom ENM along a small number of periodically reoriented normal modes (Zheng and Brooks 2006). This approach uses a small number of long-range distance constraints to specify forces that act on the ENM and thus appears well suited for modeling with EPR distance constraints.

However, it is known that not all large-scale protein motions are modeled well by ENM (Yang et al. 2007). For instance, reconfiguration of interaction networks of $\mathrm{H}$ bonds and salt bridges during the structural transition as suggested for the dopamine transporter DAT with the LeuT fold (Shan et al. 2011) is unlikely to be captured by the coarse-grained ENM. It is thus an open question whether such an approach can be applied to secondary active transporters.

In this work we analyze structural variation within and structural transition of the ten-helix core in the LeuT fold of secondary active transporters based on the set of existing crystal structures. The article is structured as follows. We start by presenting a structural alignment of the core transmembrane domains (TMDs) of the seven proteins whose structures have been solved. Based on this alignment we characterize the variability of internal conformation and position of individual TMDs. We then go on to discuss how the classification of crystal structures proposed in (Forrest et al. 2011) relates to steps in the transport cycle. We identify sets of pairwise significantly different structures of the same protein for LeuT, Mhp1, AdiC, and vSGLT. For the structural transitions within 
these sets we provide phenomenological descriptions of the motion.

Then we turn to the question whether ENMs are a useful tool for secondary transporters in the LeuT fold. We show that the core architecture is reflected in the mode covariance matrix and discuss what conclusions on collective protein motion can be drawn from this matrix. Furthermore, we test how well structural changes in the LeuT fold are characterized by a limited number of low-frequency normal modes of the ENM and whether recomputation of the modes during the structural transition improves coverage of the coordinate change. Finally we discuss what picture emerges from our results on the large-scale structural changes.

\section{Methods}

All protein visualization was performed with the opensource software package MMM, version 2011.1, which is available for free from our homepage (http://www.epr. ethz.ch/software/index). Structure superposition, computation of elastic network models and covariance matrices, and fitting were performed with MMM subroutines. Coarse-grained analysis of the structural transition in Mhp1 in terms of TMD mean axes was performed with homewritten Matlab scripts, using MMM subroutines. Scripts that are not part of MMM can be obtained from the author on request.

\section{Structural alignment}

All structure superpositions in this work were performed on the first chain reported in the PDB file if several chains of the transporter were present. Comparison of structure across different proteins requires alignment of corresponding residues. Owing to low sequence homology in the LeuT fold, such alignment cannot be achieved with standard sequence alignment tools. Instead, we opted for structural alignment of the ten core TMDs, starting from an assignment of these TMDs in the seven LeuT fold proteins with known structures given by (Schulze et al. 2010). We allowed for shifts of the TMDs by \pm 1 residue with respect to these assignments. In a set of 14 structures of the seven proteins (LeuT: PDB identifiers 2A65, 2QJU, 3F3A; Mhp1: 2JLN, 2JLO, 2X79; CaiT: 3HFX, 2WSW, 2WSX; AdiC: 3NCY, 3LRB; vSGLT: 3DH4; BetP: 2WIT; ApcT: 3GIA) we shifted the residue ranges that were assigned to the individual TMDs to minimize $\mathrm{C}^{\alpha}$ root mean square deviation (r.m.s.d.) from the reference structure 2A65, which has the best resolution. During the process, core TMD lengths were reduced if this was necessary to keep all core residues within the ranges originally assigned for the
TMDs in (Schulze et al. 2010). Thus, the alignment defines a minimal ten-helix core.

\section{Standard frame}

For the standard frame we have chosen the $z$ axis along the bundle axis, defined as a line with minimum r.m.s.d. from all $\mathrm{C}^{\alpha}$ atoms of TMDs $1,2,6$, and 7 . The $\mathrm{z}$ axis points towards the periplasm. The midpoints of the arm TMDs 5 and 10 define a line in the $y z$ plane. That way the $x$ axis is approximately in the membrane plane, connecting the bundle and hash midpoints, whereas the $z$ axis is approximately along the membrane normal. With the inward-open structure 3TT3 of LeuT, we found that the first step of this procedure did not provide a bundle axis perpendicular to the membrane. Given the poor electron density near the $\mathrm{N}$ terminus in this structure, we excluded the first two residues of TMD 1 (residues 11 and 12) from computation of the bundle axis for all LeuT structures. This exclusion did not lead to significant changes in the orientation of the bundle axis for the other LeuT structures 2A65, 3F3A, 3GJC, and 3TT1.

\section{Characteristic angles}

Angle $\theta_{B, 4}$ includes the axis of TMD 4, defined as a line with minimum r.m.s.d. from all $\mathrm{C}^{\alpha}$ atoms of the TMD, and the bundle axis (mean axis of TMDs 1, 2, 6, and 7, with the first two residues of TMD 1 excluded for LeuT structures). This angle characterizes relative orientation of hash and bundle. A similar, but distinct characteristic angle for this relative orientation was introduced in (Forrest and Rudnick 2009). To characterize orientation of the two TMDs that belong to neither hash nor bundle, we define angles $\phi_{5}$ and $\phi_{10}$ between the standard frame $x$ axis and the projection of TMD 5 and 10 mean axis, respectively, onto the $x y$ plane.

Elastic network model and covariance matrix

We implemented an anisotropic elastic network model (ANM) as described by (Bahar et al. 2010) into our modeling software MMM. For the force constants $\gamma_{i j}$ we assumed an $r^{-6}$ dependence on $\mathrm{C}^{\alpha}-\mathrm{C}^{\alpha}$ distance $r$ (Hinsen et al. 2000). For $C^{\alpha}$ atoms that are direct neighbors or next neighbors in the peptide chain, we increase this force constant by a factor of 10,000 to constrain the corresponding distances, which are fixed by peptide bond geometry. A force-field-based parametrization came to a similar result for direct neighbors (Hinsen et al. 2000), while the necessity to constrain the next neighbor distance was recognized by (Zheng and Brooks 2006), who implemented this constraint in a different way. 
Diagonalization of the Hessian matrix of the ANM provides a matrix of eigenvectors $\boldsymbol{u}$, corresponding to the normal modes of the ANM. For a model with $n \mathrm{C}^{\alpha}$ atoms, the $3 n$ eigenvectors take the form

$\boldsymbol{u}_{k}=\left(\Delta x_{1}, \Delta y_{1}, \Delta z_{1}, \Delta x_{2}, \Delta y_{2}, \Delta z_{2}, \ldots, \Delta x_{n}, \Delta y_{n}, \Delta z_{n}\right)$

where the $\Delta x_{i}$ are displacements of the $x$ coordinate of the $\mathrm{C}^{\alpha}$ atom with number $i$. From the set of eigenvectors, a perresidue covariance matrix $\boldsymbol{C}$ can be computed by rewriting $\boldsymbol{u}_{k}$ as

$\boldsymbol{u}_{k}=\left(\Delta \boldsymbol{r}_{k, 1}, \Delta \boldsymbol{r}_{k, 2}, \ldots, \Delta \boldsymbol{r}_{k, n}\right)$

where the $\Delta \boldsymbol{r}_{k, i}$ are Cartesian displacement vectors in mode $k$ for atom $i$. The matrix elements $C_{i j}$ of $\boldsymbol{C}$ are given by

$C_{i j}=\sum_{k=7}^{3 n} \frac{\Delta \boldsymbol{r}_{k, i} \cdot \Delta \boldsymbol{r}_{k, j}}{\lambda_{k}}$

where the $\lambda_{k}$ are eigenvalues of the Hessian matrix. The eigenvectors are ordered by ascending eigenvalue, and the first six eigenvectors are neglected, as they correspond to overall rotation and translation of the peptide chain.

Coverage of conformational changes by slow modes of an ANM

The coordinate change between two structures can be written as a vector $\Delta \boldsymbol{R}$ that is structured in the same way as the $\boldsymbol{u}_{k}$ in Eq. (1). With the complete matrix $\boldsymbol{u}$ of eigenvectors of the Hessian, the system of linear equations

$\Delta R=u d$

has a unique solution for the coefficient vector $\boldsymbol{d}$. In other words, the coordinate change $\Delta \boldsymbol{R}$ can be expressed as a linear combination of displacements along the normal modes of the ANM that are represented by the eigenvectors. If the two structures have been superimposed before by finding the rotation and translation that minimize the $\mathrm{C}^{\alpha}$ r.m.s.d., the first six coefficients $d_{1} \ldots d_{6}$ are exactly zero. In this case, the basis can be reduced by excluding eigenvectors $\boldsymbol{u}_{1} \ldots \boldsymbol{u}_{6}$. Furthermore, all coefficients $d_{k}$ can be taken as positive, as multiplication of an eigenvector $\boldsymbol{u}_{k}$ with -1 provides another valid set of normal modes.

With a given energy, larger displacements can be obtained along normal modes with low eigenvalues, which correspond to the eigenvectors $\boldsymbol{u}_{k}$ with small $k$. It follows that the coefficients $d_{k}$ should have a tendency to decrease with increasing index $k$.

We now consider a restricted basis $\boldsymbol{v}$ of normal modes that is constructed from the modes $k=7 \ldots B+6$ of $\boldsymbol{u}$ with $B<3 n-6$. In general, a linear combination of the $B$ modes with new coefficients $d_{k}$ will not exactly reproduce the coordinate change $\Delta \boldsymbol{R}$. We can still solve for the coefficient vector $\boldsymbol{d}$ in a least-squares sense,
$\boldsymbol{d}_{\mathrm{LSQ}}=\min _{\boldsymbol{d}}\left(\|\boldsymbol{v d}-\Delta \boldsymbol{R}\|^{2}\right)$.

The remaining r.m.s.d. $\Delta_{B, 0}$ between $\boldsymbol{v} \boldsymbol{d}$ and $\Delta \boldsymbol{R}$ can be compared to the $\mathrm{C}^{\alpha}$ r.m.s.d. between the two structures $\Delta_{\exp }$ in order to assess completeness of the reduced basis $v$ in describing the structural transition. The ratio $f_{0}=\left(\Delta_{\exp }-\right.$ $\left.\Delta_{B, 0}\right) / \Delta_{\exp }$ is a measure for the fraction of the structural change that is covered by the reduced basis of normal modes. Reduction of the basis of normal modes can be characterized by the fraction of modes $b=B /(3 n-6)$.

The solution of Eq. (5) is not necessarily the best description of the structural change that can be obtained with an ANM with $B$ modes. This is because the normal modes are computed in a harmonic approximation, which is not valid for large-scale structural changes. The problem can be reduced by scaling $\boldsymbol{d}_{\mathrm{LSQ}}$ by a factor $s$ so that $\delta \boldsymbol{R}=s \cdot \boldsymbol{v} \boldsymbol{d}_{\mathrm{LSQ}}$ corresponds to only a small coordinate change. Here we limit the maximum coordinate change of any $\mathrm{C}^{\alpha}$ atom to $0.2 \AA$. After adding $\delta \boldsymbol{R}$ to the coordinates, we compute a new Hessian and new normal modes and solve Eq. (5) again to obtain a new set of coefficients $\boldsymbol{d}_{\mathrm{LSQ}}$ for the next step. This procedure is then iterated until the coordinate set converges.

In all cases except one this algorithm converged to a final coordinate set $\boldsymbol{R}_{\mathrm{f}}$ after less than 150 iterations. We observed very slow convergence for the transition 3OB6 $\rightarrow$ 3L1L and stopped the fitting after 400 iterations. Owing to the basis reduction, the final coordinate set $\boldsymbol{R}_{\mathrm{f}}$ differs from the coordinates of the experimental end point structure. We denote the r.m.s.d. between $\boldsymbol{R}_{\mathrm{f}}$ and the experimental end point structure as $\Delta_{B, 1}$. The fractional coverage $f_{1}=\left(\Delta_{\exp }-\Delta_{B, 1}\right) / \Delta_{\exp }$ is a measure for the fraction of the structural change that is covered by the reduced basis of continuously updated normal modes. This procedure is similar in spirit to the algorithm of (Zheng and Brooks 2006), except that we drive the transition directly to the known endpoint structure instead of relying on a small number of distance constraints. This simpler problem does not suffer from potential overfitting.

\section{Results and discussion}

\section{Structural alignment}

The core alignment of all transporters in the LeuT fold that have been crystallized to date is shown in Table 1. Note that TMD numbering refers to the core, whereas in some proteins additional TMDs exist $\mathrm{N}$-terminally from the core, so that TMD numbering in the original publications on the structures may differ. For example, there is one additional $\mathrm{N}$-terminal TMD in vSGLT; thus, the core TMD 1 is TMD 2 in the structure. In our assignment for Mhp1, TMD 9 and 
10 are direct neighbors without an intervening loop. While it might be more appropriate to assign the two border residues as a short turn, our subsequent discussions are not affected by such redefinition.

The total core size is 241 TMD residues. The list of lengths of the ten TMDs $(27,23,32,17,26 / / 22,22,31,17$, 24) reflects pseudosymmetry of the two inverted repeats, which are separated here by the double slash. Although sequence homology in the LeuT fold is generally poor, we checked for any peculiarities in the sequence alignments of the individual TMDs. The only distinctive feature is a high incidence of aromatic residues near the region of TMD 6 , which is unwound in most, but not all structures in the LeuT fold (Scheme 1). This feature may be functionally relevant. Of these residues F253 in LeuT has been implied in occlusion of the periplasmic pathway to the central binding side and Y263 in vSGLT in occlusion of the cytoplasmic pathway (Abramson and Wright 2009). Furthermore, by molecular dynamics (MD) simulations rotamer changes of residues from this range were implied in changes of accessibility of the central binding site for the substrate for both LeuT (Claxton et al. 2010) and the dopamine transporter DAT, which shares the LeuT fold (Shan et al. 2011). No high incidence of aromatic residues is found near the unwound region of the pseudosymmetryrelated TMD 1 of the first repeat.

The similarity of the core architectures can be appreciated from Fig. 1, where the outward occluded structure of LeuT and the structure most distant from it (maximum core $\mathrm{C}^{\alpha}$ r.m.s.d.) are shown from a view that is approximately perpendicular to the membrane.

The $\mathrm{C}^{\alpha}$ r.m.s.d. for pairwise alignment of the cores of different proteins in the LeuT fold varies between 1.86

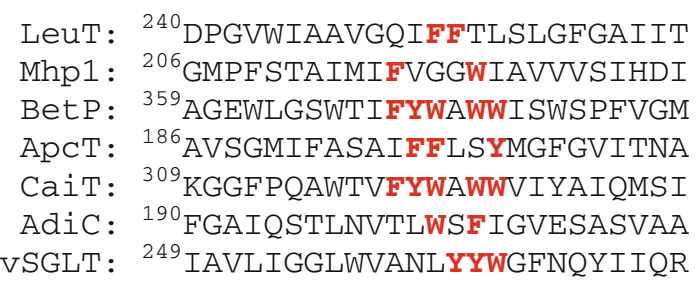

Scheme 1 Sequence alignment for core TMD 6

(CaiT: 3HFX and BetP: 2WIT, both from the BCCT family) and $5.87 \AA$ (vSGLT: 3DH4 and AdiC: 3NCY). This can be compared to the structural change between the outward-open structure of Mhp1 (2JLN) and the inwardopen structure of the same protein (2X79), which is $2.93 \AA$. The comparison suggests that the differences of core TMD internal conformation and orientation between the individual proteins are not exclusively due to idiosyncrasies of the proteins, but may be related at least partially to different conformational states of the shared architecture.

To analyze internal conformation variability of individual TMDs we have considered the mean $\mathrm{C}^{\alpha}$ r.m.s.d. for pairwise superposition of single TMDs in the set of structures 2WSW, 2WIT, 3DH4, 2A65, 2JLN, 3NCY, and 3GIA (Fig. 2). For most TMDs we find a mean $C^{\alpha}$ r.m.s.d. between 1.5 and $2.5 \AA$. The exceptions are TMD 4 with a very low internal conformation variability of only $0.57 \AA$ and TMD 6 with a very large internal conformation variability of $3.70 \AA$. Slightly enhanced internal conformation variability between 2 and $2.5 \AA$ is observed for TMDs 1,2 , 8, and 10. Based on FRET measurements and steered MD simulations, TMD 1 has been implied in the transition between the outward-facing and inward-facing state of LeuT (Zhao et al. 2010), a prediction that was later

Table 1 Alignment of core TMDs for seven secondary transporters from the LeuT fold

\begin{tabular}{|c|c|c|c|c|c|c|c|}
\hline Protein & LeuT & Mhp1 & BetP & ApcT & CaiT & AdiC & vSGLT \\
\hline TMD 1 & $11-35$ & $29-53$ & $138-162$ & $10-34$ & $88-112$ & $11-35$ & $53-77$ \\
\hline TMD 2 & $42-67$ & $59-84$ & $180-205$ & $40-65$ & $133-158$ & $41-66$ & $82-107$ \\
\hline TMD 3 & $89-120$ & $102-133$ & $234-265$ & $85-116$ & $188-219$ & $81-112$ & $126-157$ \\
\hline TMD 4 & $168-183$ & $142-157$ & $280-295$ & $125-140$ & $232-247$ & $125-140$ & $162-177$ \\
\hline TMD 5 & $191-214$ & $164-187$ & $302-325$ & $147-170$ & $255-278$ & $144-167$ & 185-208 \\
\hline TMD 6 & $240-264$ & $206-230$ & $359-383$ & $186-210$ & $309-333$ & $190-214$ & $249-273$ \\
\hline TMD 7 & $275-300$ & $249-274$ & $397-422$ & $221-246$ & $347-372$ & $224-249$ & $282-307$ \\
\hline TMD 8 & $336-367$ & $294-325$ & $449-480$ & $268-299$ & $403-434$ & $274-305$ & $346-377$ \\
\hline TMD 9 & 379-394 & $340-355$ & $492-507$ & $321-336$ & $449-464$ & $327-342$ & 399-414 \\
\hline TMD 10 & $401-424$ & $356-379$ & $515-538$ & $340-363$ & $469-492$ & $351-374$ & $424-447$ \\
\hline $\mathrm{PDB}^{\mathrm{a}}$ & $2 \mathrm{~A} 65$ & $2 J L N$ & 2WIT & 3GIA & 3HFX & $3 \mathrm{NCY}$ & 3DH4 \\
\hline $\mathrm{C}^{\alpha}$ r.m.s.d. $(\AA)^{\mathrm{b}}$ & 0.0 & 3.28 & 3.71 & 3.83 & 3.87 & 4.02 & 4.43 \\
\hline
\end{tabular}

${ }^{\text {a }}$ Entry of a representative structure

${ }^{b}$ With respect to LeuT structure 2A65. For proteins with several structures, the number corresponds to the representative structure 
Fig. 1 Core architecture of LeuT structure 2A65 (a) and vSGLT structure 3DH4 (b). TMDs are color coded with numbering starting with the first core TMD. Collectively moving groups of TMDs are marked as bundle and hash motif in (b)
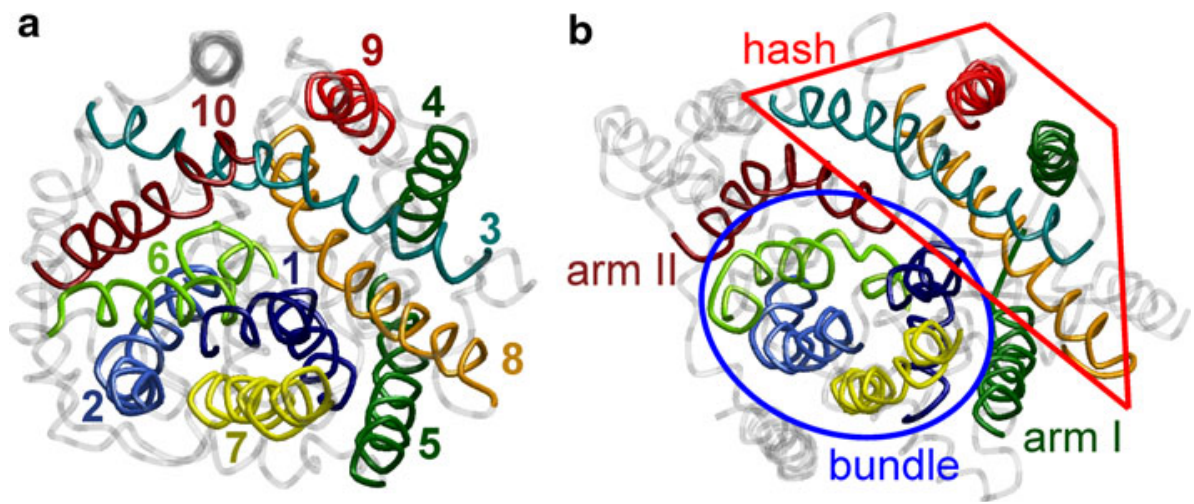

confirmed by a crystal structure for an inward-facing state (Krishnamurthy and Gouaux 2012).

Comparison of the outward occluded structure of LeuT (2A65), an occluded structure of BetP (2W8A, meanwhile superseded by 2 WIT), and the inward-open structure of vSGLT (3DH4) suggested that TMD 8 changes from a kinked internal conformation via a slightly kinked internal conformation to a straight TMD in the outward-open to inward-open transition (Ressl et al. 2009). For the apo form of prolin/sodium symporter PutP, a helix-loop-helix model based on EPR data revealed a kink of TMD 8 very similar to the one seen in LeuT (Hilger et al. 2009), whereas the same TMD is straight in inward-open conformations of vSGLT, which belongs to the same protein family of sodium substrate symporters (SSS) as PutP. However, in Mhp1 and LeuT, which could be crystallized in outwardopen and inward-open structures, only slight straightening is observed (vide infra). In the SLC 7 family of amino acid transporters TMD 8 is straight in the outward-open

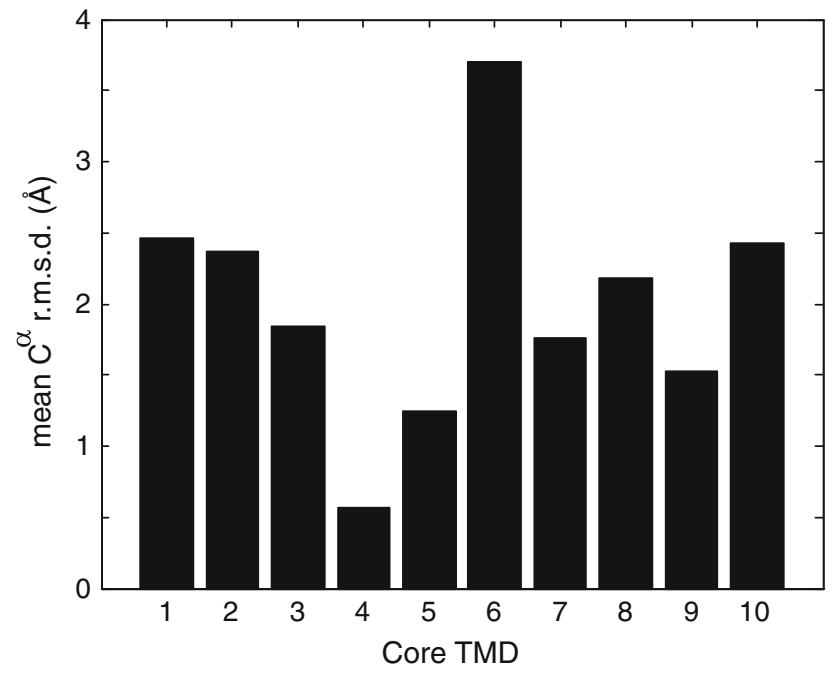

Fig. 2 Conformational variability of core TMDs in the LeuT fold characterized by the mean r.m.s.d. for pairwise superposition of the $\mathrm{C}^{\alpha}$ atoms of each individual TMD in the structure space with PDB identifiers 2WSW, 2WIT, 3DH4, 2A65, 2JLN, 3NCY, and 3GIA structure of the arginine/agmatine antiporter AdiC (3NCY) and kinked in the inward-open structure of ApcT (3GIA). Hence, even if a change of internal conformation of TMD 8 is a universal feature of the transition from the outwardopen to the inward-open state, extent and direction of this change differ between different proteins for currently unknown reasons.

We have taken advantage of the small internal conformation variability of TMD 4 to define a robust parameter $\theta_{B, 4}$ (see Sect. "Methods") that characterizes relative orientation of the bundle of TMDs 1, 2, 6, and 7 and the hash motif made up by TMDs $3,4,8$, and 9 (see Fig. 1b). Such a parameter is of interest as motion of the bundle relative to scaffold TMDs (Forrest et al. 2008) or to the hash motif (Shimamura et al. 2010) was suggested as the main conformational change in the outward-open to inward-open structural transition. In structures that were previously assigned as outward-open or outward-occluded (Ce, CSe, CSec) angle $\theta_{B, 4}$ is larger than $26^{\circ}$ with typical values around $30^{\circ}$ (Table 2). The only exception is the CSec conformation of the antiporter AdiC (3L1L), which has $\theta_{B, 4}=19.2^{\circ}$. For all inward-open structures, we find $\theta_{B, 4}<18^{\circ}$ with typical values around $15^{\circ}$. The BetP structure 2WIT, which is assigned as CSic, but was originally assigned as $\mathrm{CSc}$, has $\theta_{B, 4}=17.4^{\circ}$.

Relation between crystalline conformations and functional states

Recently a classification was suggested for secondary active transporter conformations encountered in crystal structures (Forrest et al. 2011). In this classification (Scheme 2a) the outward-open to inward-open transition starts from the Ce conformation, where no substrate or ions are bound, and the central binding site is fully accessible from the outside and inaccessible from the inside. It proceeds via the CSe conformation, where substrate and ion(s) are bound and the central binding site is still fully accessible from the outside to the CSec conformation, where the central binding site becomes weakly occluded. 
Table 2 Coarse-grained characteristics of conformations in the LeuT fold

\begin{tabular}{|c|c|c|c|c|c|c|c|c|c|}
\hline PDB & $3 \mathrm{HFX}$ & 2WSX & $2 \mathrm{WIT}$ & 3DH4 & 2XQ2 & 3TT1 & $3 \mathrm{~F} 3 \mathrm{~A}$ & $2 \mathrm{~A} 65$ & $3 \mathrm{GJC}$ \\
\hline Protein & CaiT & CaiT & BetP & vSGLT & vSGLT & LeuT $^{\mathrm{a}}$ & LeuT $^{\mathrm{a}}$ & LeuT $^{\mathrm{a}}$ & LeuT $^{\mathrm{a}}$ \\
\hline Conformation & $\mathrm{CSi}$ & $\mathrm{CSi}$ & $\mathrm{CSic}$ & $\mathrm{CSic}$ & $\mathrm{Ci}$ & $\mathrm{Ce}$ & $\mathrm{CSe}$ & $\mathrm{CSec}$ & S2 \\
\hline$\theta_{B, 4}\left(^{\circ}\right)$ & 16.1 & 16.3 & 17.4 & 11.5 & 17.7 & 33.3 & 33.0 & 29.0 & 29.9 \\
\hline$\phi_{5}\left(^{\circ}\right)$ & 12.9 & 15 & 27.7 & 9.5 & 2.1 & 38.5 & 38.2 & 33.1 & 30.6 \\
\hline$\phi_{10}\left({ }^{\circ}\right)$ & 30.1 & 28.1 & 24.8 & 45.6 & 48.3 & 10.9 & 10.8 & 15.8 & 14.1 \\
\hline PDB & 3TT3 & $2 \mathrm{JLN}$ & & & & 3GIA & 3LRB & $30 B 6$ & 3L1L \\
\hline Protein & LeuT $^{\mathrm{a}}$ & Mhp1 & & & & ApcT & AdiC & AdiC & AdiC \\
\hline Conformation & $\mathrm{Ci}$ & $\mathrm{Ce}$ & & & & $\mathrm{Cic}$ & $\mathrm{Ce}$ & $\mathrm{CSe}$ & $\mathrm{CSec}$ \\
\hline$\theta_{B, 4}\left(^{\circ}\right)$ & 12.5 & 29.3 & & & & 16.2 & 26.7 & 28.2 & 19.2 \\
\hline$\phi_{5}\left(^{\circ}\right)$ & 0.1 & 33.5 & & & & 5.7 & 21.5 & 18.0 & 19.9 \\
\hline$\phi_{10}\left(^{\circ}\right)$ & 25.6 & 11.8 & & & & 26.7 & 15.2 & 16.1 & 25.1 \\
\hline
\end{tabular}

Conformation assignments were taken from (Forrest et al. 2011), except for 2XQ2, 3TT1, 3TT3, and 3OB6, which were assigned analogously. $\mathrm{S} 2$ is an outward-open structure with a putative secondary binding site blocked

${ }^{\text {a }}$ For LeuT, the first two residues of TMD1 were excluded from computation of the bundle axis

The pivot point of the transition is the CSc conformation where the central binding site is strongly occluded with respect to both the outside and inside. This conformation then converts to CSic, where the binding site is only weakly occluded to the inside, but inaccessible from the outside. From the inside-open conformation CSi substrate and ion(s) can dissociate to give the inside-open apo conformation $\mathrm{Ci}$. In symporters, $\mathrm{Ci}$ must be able to convert back to $\mathrm{Ce}$ by thermal excitation to close the cycle, whereas in antiporters this conversion must be forbidden to avoid uncoupling of substrate and ion transport.

From a functional point of view the succession of states is slightly different. To minimize uncoupled transport, in symporters the ion must bind to the transporter before the substrate ("first on") and must also unbind before the substrate ("first off") (Forrest et al. 2011). We neglect intermediate transition states, except for the occluded state TISo where the central binding site is inaccessible from both the outside and inside. In this picture (Scheme 2b) the outward-open apo state Se first binds the ion to give SIe and then the substrate to give SISe. This state converts via the transition state TISo to an inward-open, substrate and ionbound state SISi, which first loses the ion to give SSi and then the substrate to give the inward-open apoprotein $\mathrm{Si}$.

As pointed out by (Mchaourab et al. 2011) and as is known from NMR studies on soluble enzymes (HenzlerWildman and Kern 2007), functional states are not necessarily associated with well-defined conformations. Such

$$
\begin{aligned}
& \text { a } \mathrm{Ce} \leftrightarrow \mathrm{CSe} \leftrightarrow \mathrm{CSec} \leftrightarrow \mathrm{CSc} \leftrightarrow \mathrm{CSic} \leftrightarrow \mathrm{CSi} \leftrightarrow \mathrm{Ci} \\
& \text { b } \mathrm{Se} \leftrightarrow \mathrm{SIe} \leftrightarrow \mathrm{SISe} \leftrightarrow \mathrm{TISo} \leftrightarrow \mathrm{SISi} \leftrightarrow \mathrm{SSi} \leftrightarrow \mathrm{Si}
\end{aligned}
$$

Scheme 2 Secondary transporter conformations (a) and functional states $(\mathbf{b})$ states may correspond to ensembles of conformations, and state changes may be associated with shifting weights between distinct subensembles. Despite this complication, structural changes between the conformations indicated in Scheme $2 \mathrm{a}$ are of interest for understanding the functional states. These changes reveal which parts of the structure are flexible and which parts move collectively. This is still true for conformations that may be off-path because of blocking of a binding site by detergent, as suggested for structure 2A65 of LeuT (Quick et al. 2009) but dismissed by (Wang et al. 2012), or downregulated because of low osmolarity, as suggested for structure 2WIT of BetP (Forrest et al. 2011). In the following, we thus turn to an analysis of structural changes.

Identification of distinct protein conformations in the LeuT fold

For identification of structural transitions we originally considered all protein structures in the LeuT fold that were published in the PDB by the end of 2011. Of the 23 structures of LeuT bound to different substrates and inhibitors, 18 structures are within $0.3 \AA \mathrm{C}^{\alpha}$ r.m.s.d. from the best resolved structure 2A65. Structures 3QS5 and 3QS6 differ by only 0.45 and $0.5 \AA$ from $2 \mathrm{~A} 65$ and by only $0.26 \AA$ from each other. This group of 20 structures was assigned to the CSec conformation. For the remaining three

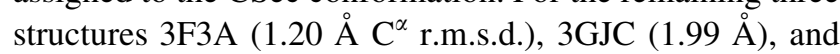
3QS4 (1.20 $\AA$ ), we computed pairwise $C^{\alpha}$ r.m.s.d. of the core, including TMDs and intervening loops. This revealed that structure $3 \mathrm{~F} 3 \mathrm{~A}$ with the competitive inhibitor tryptophan bound in a second binding site and structure 3QS4 of mutant F259V also bound to tryptophan agree within $0.24 \AA$. These structures represent a CSe conformation. 
Structure 3GJC of mutant E290S with $n$-octyl- $\beta$-d-glucopyranoside (OG) bound in the same site deviates by $1.10 \AA$ from these structures and was assigned as a conformation with blocked secondary binding site $\mathrm{S} 2$ by (Forrest et al. 2011)

While this work was under review, a number of new LeuT structures appeared. The first group of these structures addressed the problem of possible OG binding and other influence of detergents in the structures previously assigned to the CSec state by growing crystals from bicelles and using a selenium-containing analog of $\mathrm{OG}$ (Wang et al. 2012). In the second work, rationally designed mutants and complexation with antibody fragments provided, among others, the inward-open structure 3TT3 ( $\mathrm{Ci}$ state) and an outward-open substrate-free structure 3TT1 (Ce state) (Krishnamurthy and Gouaux 2012). The core of structure 3TT1 deviates by only 0.42 and $0.50 \AA$ from the core of structures $3 \mathrm{QS} 4$ and $3 \mathrm{~F} 3 \mathrm{~A}$ (CSe state), respectively, a fact already noticed by Krishnamurthy and Gouaux. Structure 3TT3 deviates by $3.70,3.34$, and $3.16 \AA$ from $3 \mathrm{~F} 3 \mathrm{~A}, 3 \mathrm{GJC}$, and $2 \mathrm{~A} 65$, respectively. For all the remaining new LeuT structures the core deviation from 2A65 (CSec state) does not exceed $0.49 \AA$. All structures obtained by crystallization from bicelles agree better with each other (maximum deviation $0.18 \AA$ ) than with structure $2 \mathrm{~A} 65$ (typical deviation $0.48 \AA$ ), except for structure 3USI. For LeuT we shall thus consider structural transitions between 3TT1, 3F3A, 2A65, 3GJC, and 3TT3, which are the best resolved structures in their respective groups.

The three Mhp1 structures 2JLN, 2JLO, and 2X79 are all distinct with $\mathrm{C}^{\alpha}$ r.m.s.d. of $1.13 \AA$ between $2 \mathrm{JLN}$ and $2 \mathrm{JLO}$ and $3.03 \AA$ between $2 \mathrm{JLO}$ and 2 X79. They combine to a succession of conformations $\mathrm{Ce} \leftrightarrow \mathrm{CSec} \leftrightarrow \mathrm{Ci}$.

The two BetP structures 2WIT with substrate glycine betaine bound and 3P03 of mutant G153D with choline bound have a core $\mathrm{C}^{\alpha}$ r.m.s.d. of $1.04 \AA$. The significant deviations are confined to the loops between TMDs. As loop structure is easily influenced by crystal packing, we refrain from further study of the difference between these two structures.

The three ApcT structures 3GI8, 3GI9, and 3GIA have mutual $\mathrm{C}^{\alpha}$ r.m.s.d. of less than $0.5 \AA$, which is insignificant at their resolution. The two CaiT structures $3 \mathrm{HFX}$ and 2WSX of the same protein differ by $1.43 \AA$. However, as in the case of BetP, significant differences are strictly confined to the loops. Hence, we also refrain from discussing structural changes in ApcT and CaiT.

In contrast, the five AdiC structures 3LRB (apo), 3LRC (apo), 3L1L (arginine bound), 3NCY (apo complexed with a Fab fragment), and 3OB6 (mutant N101A arginine bound in an open-to-outward conformation) are pairwise significantly different with $\mathrm{C}^{\alpha}$ r.m.s.d. larger than $2 \AA$, except for the pair 3LRB/3LRC, which corresponds to the same conformation. Structures $3 \mathrm{LRB}$ and $3 \mathrm{NCY}$ can be assigned to different $\mathrm{Ce}$ conformations, $3 \mathrm{OB} 6$ to a CSe conformation, and $3 \mathrm{~L} 1 \mathrm{~L}$ to a $\mathrm{CSec}$ conformation. We shall consider the sequence of structural transitions $3 \mathrm{LRB} \leftrightarrow 3 \mathrm{OB} 6 \leftrightarrow 3 \mathrm{~L} 1 \mathrm{~L}$ as well as the transition $3 \mathrm{LRB} \leftrightarrow 3 \mathrm{NCY}$.

Finally, the cores of the galactose-bound structure (3DH4) and the apo structure (2XQ2) of vSGLT differ by $2.19 \AA$ A. These structures can be assigned as CSic and $\mathrm{Ci}$ conformations. Note that a computational study has assigned structure $3 \mathrm{DH} 4$ as an ion-releasing state ( $\mathrm{Li}$ and Tajkhorshid 2009). In the nomenclature of Scheme $2 b$ this structure thus corresponds to an SSi rather than an SISi state.

Coarse-grained analysis of the $\mathrm{Ce} \leftrightarrow \mathrm{Ci}$ transition in Mhp1

The only protein for which both an outward-open (2JLN) and inward-open structure (2X79) was known on initial submission of this work was Mhp1. The transition has been characterized as mainly a relative motion of the hash motif (TMDs 3, 4, 8, and 9) with respect to the bundle (TMDs 1, 2,6 , and 7), accompanied by some bending and flexing of the arm TMDs 5 and 10 (Shimamura et al. 2010). To characterize this movement we have superimposed the bundle TMDs by minimization of $\mathrm{C}^{\alpha}$ r.m.s.d. $(0.58 \AA)$. After such superposition the $C^{\alpha}$ r.m.s.d. of the hash motif is $6.16 \AA$. The difference in orientation of the hash motif can be appreciated from the visualization in the standard frame in Fig. 3a, where TMDs are represented as sticks oriented along the mean axis of the $\mathrm{C}^{\alpha}$ atoms.

Optimum superposition of the hash motif from the outward-open structure $2 \mathrm{JLN}$ to the inward-open structure 2 X79 can be achieved by a screw transformation (minimum $\mathrm{C}^{\alpha}$ r.m.s.d. $0.89 \AA$ ). A unit vector along the screw axis has polar angles $\theta=52.1^{\circ}$ and $\phi=77.6^{\circ}$ in the standard frame (green arrow in Fig. 3b, d). The screw transformation is a rotation by $31.5^{\circ}$ about this axis, followed by a $1.9 \AA$ translation along the axis. As is apparent from Fig. 3a, the remaining deviation after this screw transformation is largely confined to TMD 3.

In the coarse-grained representation, where each TMD is represented by only its mean axis, movement of the arms can be considered as a rotation about an axis intersecting the TMD midpoints. Arm I (TMD 5) rotates by $37.7^{\circ}$ about an axis that includes an angle of $67.6^{\circ}$ with the screw axis of the hash motif, whereas arm II (TMD 10) rotates by $19.5^{\circ}$ about an axis that includes an angle of $39.5^{\circ}$ with the screw axis. The view along the screw axis (Fig. 3a, b) shows that this rotation partially follows the hash rotation. The view from the periplasmic side along the membrane normal (Fig. 3d) demonstrates that the arms rotate more 

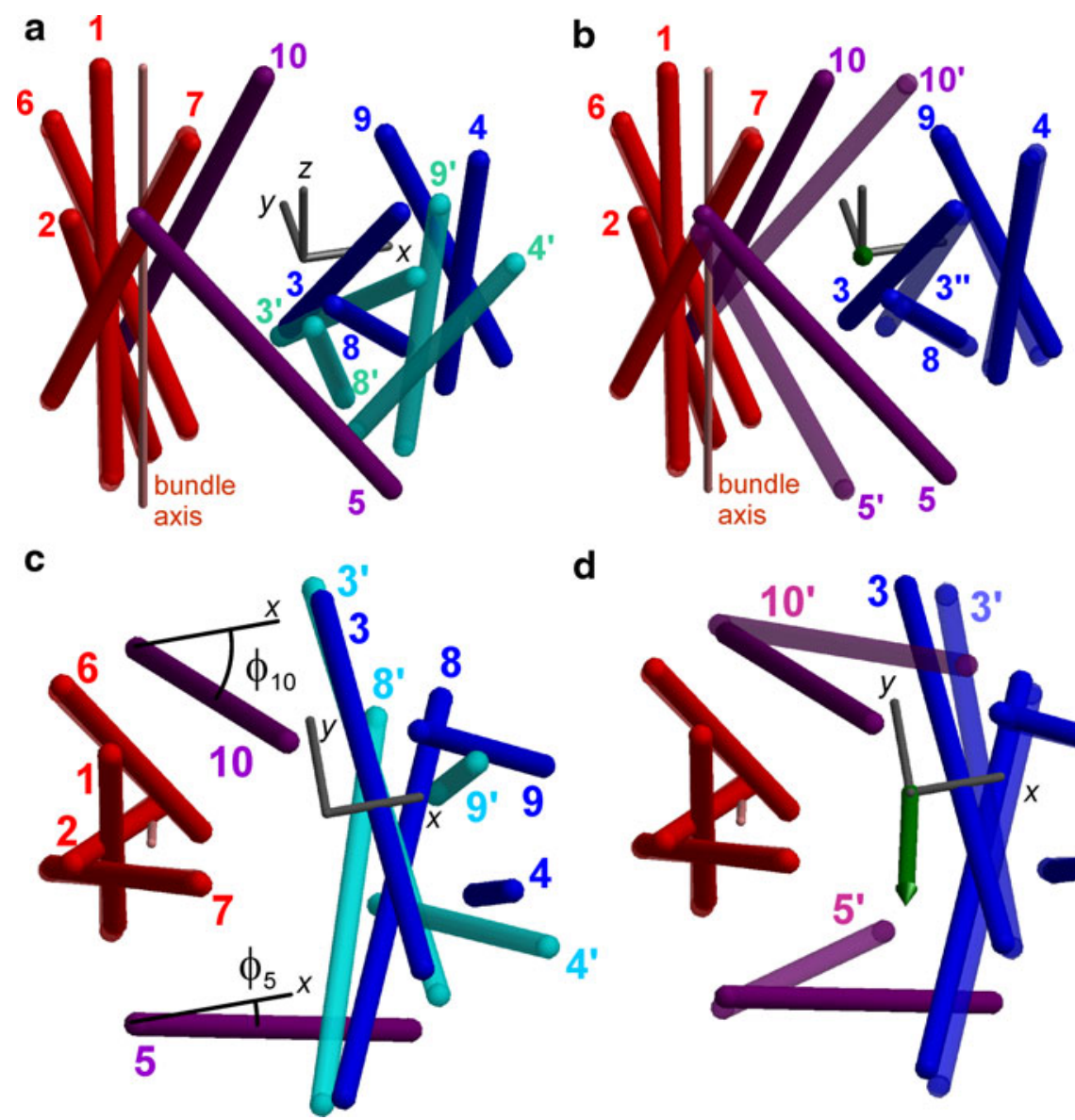

d

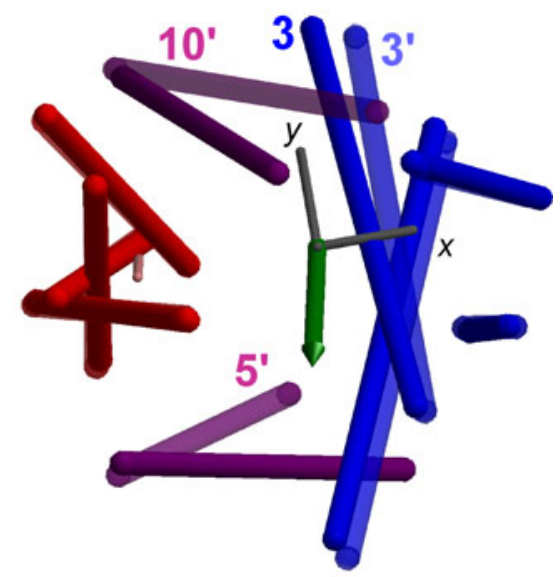

Fig. 3 Coarse-grained visualization of the ten-helix core in Mhp1 structures 2X79 (inward open, solid red, blue, and purple) and 2JLN (outward open, transparent red and cyan). a Structures are superimposed on bundle TMDs $1,2,6$, and 7 ( $C^{\alpha}$ r.m.s.d. $0.58 \AA$ ). The $C^{\alpha}$ r.m.s.d. between the two hash motifs (blue and cyan) is $6.16 \AA$. Coupler TMDs 5 and 10 are not displayed for structure 2JLN. The standard frame is visualized as a grey tripod (see text). The screw axis for the hash motif movement points to the observer. b A screw

transformation was applied to the hash motif of 2JLN, resulting in the position and orientation shown as transparent blue sticks. Coupler TMDs $5^{\prime}$ and $10^{\prime}$ of structure $2 \mathrm{JLN}$ are shown as transparent purple sticks. The transformed hash motif superimposes with $\mathrm{C}^{\alpha}$ r.m.s.d. of $0.89 \AA$ onto the one of structure 2 X79 (solid blue sticks). c View from the periplasmic side corresponding to (a). d View from the periplasmic side corresponding to (b). The screw axis is visualized by a green arrow

strongly than the hash about the membrane normal. Such movement may help to occlude one side of the substrate translocation pathway while opening up the other side.

If the same analysis is applied to the $\mathrm{Ce} \leftrightarrow \mathrm{CSec}$ transition between structures $2 \mathrm{JLN}$ and 2JLO, only arm II rotates by $14.9^{\circ}$. The screw transformation of the hash and any internal changes in the bundle and hash are insignificant in this transition. Note however that structure 2JLO is based on 2JLN with remodeling of only part of loop L9-10 and TMD 10 (Weyand et al. 2008).

We now discuss reorientation of the arms characterized by angles $\phi_{5}$ and $\phi_{10}$ (Fig. 3c). We have checked that this motion is generally better described as reorientation of the corresponding TMD as a whole than as an independent movement of one moiety of a kinked TMD, in agreement with the finding that the entire TMD 5 contributes to the cyoplasmic thin gate and the entire TMD 10 to the

periplasmic thick gate (Shimamura et al. 2010). Note however that a small contribution from a change in kink angle may be contained in $\phi_{5}$ and $\phi_{10}$. For inward-open conformations we find $\phi_{5}<15^{\circ}$, for outward-open conformations of symporters $\phi_{5}>26^{\circ}$ with larger values for less occluded states. The conformation of BetP assigned either as CSic or CSc (2WIT) has $\phi_{5}=27.7^{\circ}$. Outwardopen conformations $\mathrm{Ce}, \mathrm{CSe}$, and $\mathrm{CSec}$ of the antiporter AdiC have $\phi_{5} \approx 20^{\circ}$. The situation is somewhat less clear cut with respect to $\phi_{10}$. For most outward-open conformations we find $\phi_{10}<18^{\circ}$, and for all inward-open conformations we find $\phi_{10}>25^{\circ}$, with typical values of $35^{\circ}$. The conformation of BetP in between CSic and CSc (2WIT) has $\phi_{10}=24.8^{\circ}$. However, the outward-open CSec conformation of Mhp1 (2JLO) has $\phi_{10}=30.7^{\circ}$, which suggests that $\phi_{10}$ is more strongly correlated to occlusion of the extracellular path rather than to opening of 
the intracellular path. The CSec conformation of AdiC (3L1L) has $\phi_{10}=25.1^{\circ}$, again suggesting that a change in $\phi_{10}$ (reorientation of TMD 10) is mainly related to occlusion of the periplasmic pathway and uncoupled from changes in $\phi_{5}$.

Phenomenological analysis of structural transitions

Mhpl

For the transition Ce (2JLN) $\rightarrow$ CSec (2JLO) the conformation change is largely restricted to TMD 10, which kinks at the $\mathrm{N}$ terminal end (periplasmic side) as visualized in Fig. 4b. Complete visualization of the transition in Online Resource 1, page S 1, shows that TMD 10 kinks towards the bundle, thus occluding periplasmic access to the central binding site, as was already pointed out in (Weyand et al. 2008). Among the characteristic angles, $\theta_{B, 4}$ and $\phi_{5}$ only slightly decrease by 2.6 and $4.2^{\circ}$, respectively, corresponding to only $13-15 \%$ of the total change between the $\mathrm{Ce}$ and $\mathrm{Ci}$ conformations (19.7 and $28.3^{\circ}$, respectively, for the structure pair 2JLN/2X79). In contrast, $\phi_{10}$ increases by $18.9^{\circ}$, which is approximately $50 \%$ of the change between the $\mathrm{Ce}$ and $\mathrm{Ci}$ conformations. The kink or bending angle $\beta_{10}$ between the mean axes through the $\mathrm{N}$-terminal and C-terminal halves of TMD 10 increases by about $6^{\circ}$.
Analogously defined angles for TMDs 1,6 , and $5\left(\beta_{1}, \beta_{5}\right.$, $\beta_{6}$ ) change by less than $3^{\circ}$.

The transition CSec (2JLO) $\rightarrow \mathrm{Ci}$ (2X79) is strongly dominated by the reorientation of the hash motif with respect to the bundle. In addition, the kinks of TMD 8 and 10 slightly decrease (Fig. 4a, b). This straightening of TMD 8 is in line with comparison of structures of different symporters in outward-open and inward-open conformations. However, in the case of Mhp1 such straightening is not complete. Among the loops, L6-7 and L7-8 move away from the bundle axis to the outside with L7-8 simultaneously moving from the cytosol towards the membrane. The characteristic angle changes are a decrease of $17.1^{\circ}$ for $\theta_{\mathrm{B}, 4}$, a decrease of $24.1^{\circ}$ for $\phi_{5}$, and an increase of $17.6^{\circ}$ for $\phi_{10}$. Angle $\beta_{10}$ increases by $8^{\circ}$, whereas $\beta_{1}, \beta_{5}$, and $\beta_{6}$ change by less than $3^{\circ}$.

\section{LeuT}

For the Ce (3TT1) $\rightarrow$ CSe (3F3A) transition, TMD internal conformations are conserved within a $\mathrm{C}^{\alpha}$ r.m.s.d. of about $0.4 \AA$. The smallest change of $0.12 \AA$ is found for TMD 4 and the largest change of $0.43 \AA$ for TMD 6. Angles $\theta_{B, 4}$, $\phi_{5}$, and $\phi_{10}$ do not change significantly (less than $0.5^{\circ}$ ). There are no significant changes in the kink and bending angles $\beta_{k}$.
Fig. 4 Changes of TMD internal conformations for a TMD 8 of Mhp1, b TMD 10 of Mhp1, c TMD 1 of LeuT, d TMD 8 of LeuT, e TMD 1 of AdiC, $\mathbf{f}$ TMD 2 of AdiC, g TMD 6 of AdiC, $\mathbf{h}$ TMD 1 of vSGLT. Color code relates to assigned conformations: $\mathrm{Ce}$ blue, CSe cyan, CSec green, CSic magenta, Ci red. The $\mathrm{N}$ and $\mathrm{C}$ terminus of the TMDs are marked
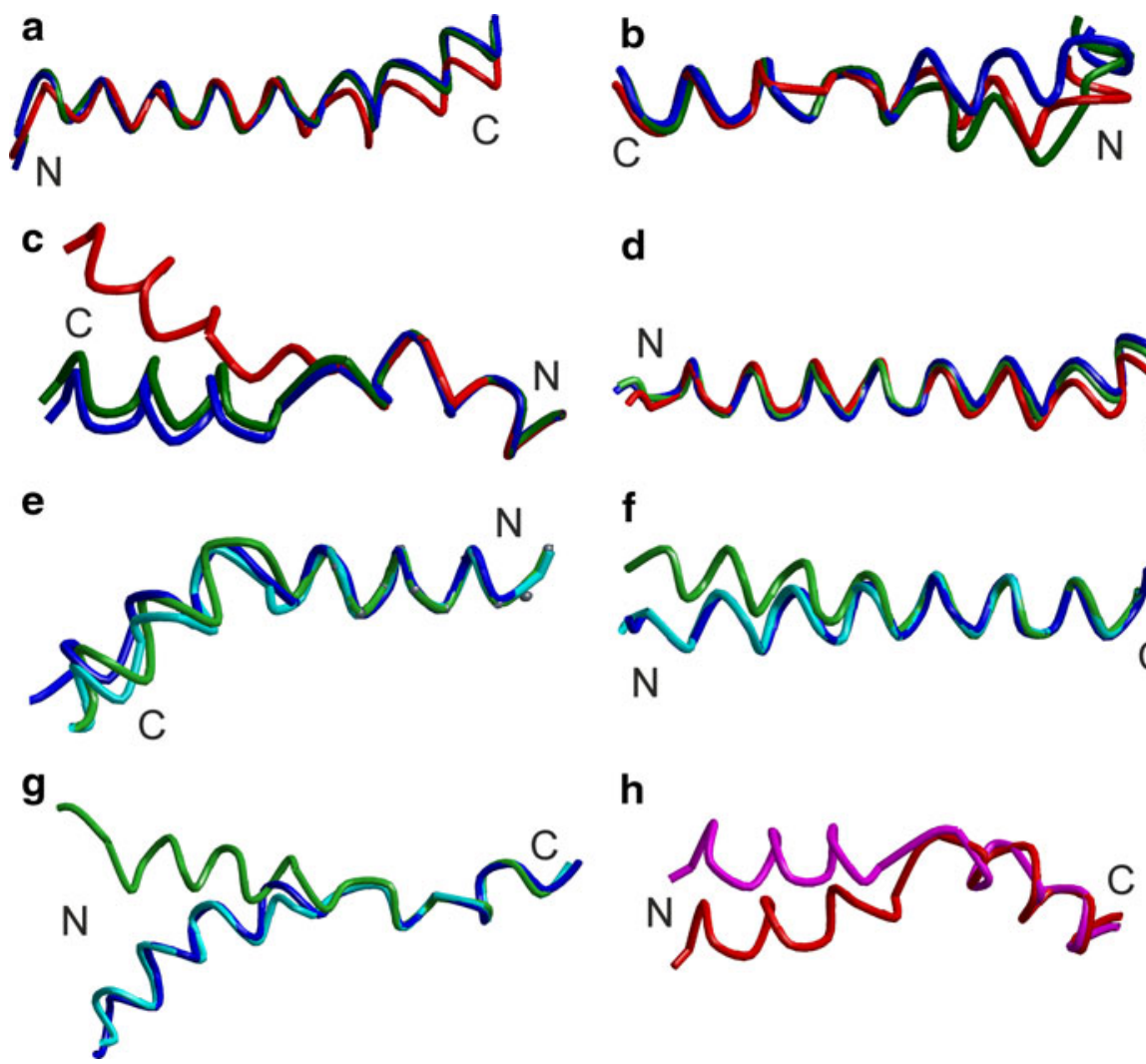

f

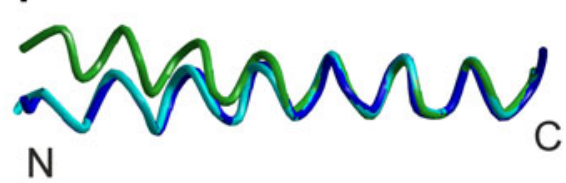

h

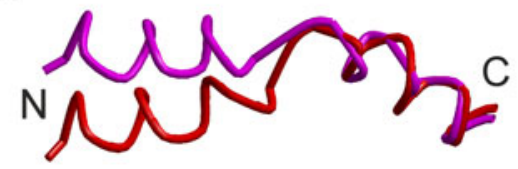


Slightly larger changes are observed for the CSe $(3 \mathrm{~F} 3 \mathrm{~A}) \rightarrow \mathrm{CSec}(2 \mathrm{~A} 65)$ transition, although the trend is the same. TMD internal conformations are conserved within a $\mathrm{C}^{\alpha}$ r.m.s.d. of $0.6 \AA$. The smallest changes of 0.09 and $0.60 \AA$ are again found for TMD 4 and 6, respectively, in agreement with findings on internal conformation variability of TMDs across different proteins in the LeuT fold. Although TMD 1 and 2 do not significantly kink, their periplasmic ends tilt somewhat away from the bundle axis towards the hash, which contributes to the occlusion of the central binding site (Online Resource 1, page S 3). This was already pointed out in (Singh et al. 2008). The kink angle $\beta_{1}$ decreases by $19.4^{\circ}$.

For the CSec (2A65) $\rightarrow$ S2 (3GJC) transition, TMD internal conformations are even better preserved. Indeed, the two structures differ only slightly except for loop L3-4. Changes of the characteristic angles and kink or bending angles do not exceed $2.5^{\circ}$.

The largest changes are observed during the CSec $(2 \mathrm{~A} 65) \rightarrow \mathrm{Ci}$ (3TT3) transition. Internal conformation of TMD 1 changes drastically by $1.68 \AA$, as visualized in Fig. 4c. Note that this change was predicted on the basis of atomistic MD simulations (Zhao and Noskov 2011). Significant conformation change is also observed for TMD 8 (0.68 $\AA$ ), whereas all other TMDs maintain conformation within $0.5 \AA \mathrm{C}^{\alpha}$ r.m.s.d., with TMD 4 again exhibiting the smallest change. Similarly to the CSec $(2 \mathrm{JLO}) \rightarrow \mathrm{Ci}$ (2X79) transition of Mhp1, TMD 8 slightly straightens, but to an even smaller extent (Fig. 4d). Also in analogy to the $\mathrm{CSec} \rightarrow \mathrm{Ci}$ transition of Mhp1, $\theta_{B, 4}$ decreases by $16.5^{\circ}$ (17.1 ${ }^{\circ}$ for Mhp1). We have checked whether the change in $\theta_{B, 4}$ is significantly affected by the conformational change of TMD 1. For that, we defined the bundle axis from only TMD 1b (residues 26-35), 2, 6, and 7. Based on this definition, angle $\theta_{\mathrm{B}, 4}^{\prime}$ differs only slightly from angle $\theta_{B, 4}$ and decreases by $14.4^{\circ}$. We conclude that the rocking bundle motion makes a significant contribution to the $\mathrm{CSec} \rightarrow \mathrm{Ci}$ transition of LeuT. Kink angles $\beta_{1}$ and $\beta_{6}$ increase by $18.5^{\circ}$ and $6.1^{\circ}$, respectively.

Likewise, the decrease in $\phi_{5}$ by $24.1^{\circ}$ observed in the the $\mathrm{CSec} \rightarrow \mathrm{Ci}$ transition of Mhp1 finds an analogy in a decrease of $\phi_{5}$ by $33^{\circ}$ in the same transition in LeuT and the increase of $\phi_{10}$ by $17.6^{\circ}$ in Mhp1 in an increase by $9.8^{\circ}$ in LeuT. These parameters, which were defined before the $\mathrm{Ci}$ structure of LeuT was published, thus appear to describe common behavior of different proteins within the LeuT fold.

AdiC

In general, outward-open states of AdiC exhibit stronger variability of TMD internal conformations than outwardopen states of Mhp1 and LeuT. In the Ce (3LRB) $\rightarrow$ CSe
(3OB6) transition, the periplasmic ( $\mathrm{C}$ terminal) moiety of the partially unwound TMD 1 slightly kinks to effect a motion of L1-2 away from the hash motif (Fig. 4e and Online Resource 1, page S6). This corresponds to an opening up of the path to the central binding site. The periplasmic ( $\mathrm{N}$ terminal) end of TMD 8 may also slightly kink away from the periplasmic path to the binding site (not shown), although this slight change is probably within uncertainty of the crystal structures. Likewise, the sections of L7-8 that are resolved in both structures move away from this path. Changes of the characteristic angles do not exceed $3.5^{\circ}$, except for kink angle $\beta_{1}$, which increases by $7.2^{\circ}$.

The structural changes are more dramatic for the CSe $(3 \mathrm{OB} 6) \rightarrow \mathrm{CSec}$ (3L1L) transition. TMDs 2 and 6 straighten (Fig. 4f, g), leading to a move of their $\mathrm{N}$ terminal ends into the periplasmic path to the central binding side (Online Resource 1, page S7). A significant relative movement of bundle and hash occurs, as indicated by a $9^{\circ}$ decrease of angle $\theta_{B, 4}$. Likewise, angle $\phi_{10}$ increases by $9^{\circ}$. In this transition, TMDs bend or kink significantly. Kink angle $\beta_{6}$ decreases by $16.3^{\circ}$, and the angles between the $\mathrm{N}$ - and C-terminal halves of TMDs 5 and 10 change by $\Delta \beta_{5}=-8^{\circ}$ and $\Delta \beta_{10}=9.2^{\circ}$. Differences between the two Ce conformations (3LRB and $3 \mathrm{NCY}$ ) are mainly confined to loop regions.

\section{vSGLT}

The CSic (3DH4) $\rightarrow$ CSi (2XQ2) transition is manifest mainly in an outward kink of the cytoplasmic ( $\mathrm{N}$ terminal) moiety of TMD 1, which leads to an opening of the cytoplasmic path to the central binding site (Fig. 4h). A slight internal conformation change of TMD 8 may also contribute to this opening, although this change may hardly exceed uncertainty of the x-ray structures (not shown). Angle $\theta_{B, 4}$ increases slightly by $6.2^{\circ}$, although generally an opening up of the cytoplasmic path is associated with a decrease of this angle. Angle $\phi_{5}$ slightly decreases and angle $\phi_{10}$ slightly increases, which is in line with expectations for an opening of the cytoplasmic path. The transition involves significant changes in kink or bending angles $\Delta \beta_{1}=-8.7^{\circ}, \Delta \beta_{5}=8.5^{\circ}$, and $\Delta \beta_{6}=5.5^{\circ}$.

Interpretation of the ANM covariance matrix

To test the predictive power and model quality of the ANM for structural transitions in the LeuT fold, we computed covariance matrices for all significantly different structures. From Fig. 5 it is clear that the common core architecture of the LeuT fold leads to common correlation features in the covariance matrix. The most obvious features correspond to collective motion within the bundle and 
Fig. 5 Per-residue covariance matrices of anisotropic network models for the core of proteins with the LeuT fold. a Mhp1 structures $2 \mathrm{JLN}$ in the Ce conformation (upper left half) and $2 \times 79$ in the $\mathrm{Ci}$ conformation (lower right half). b LeuT structures 3TT1in the Ce conformation (upper left half) and 3TT3 in the $\mathrm{Ci}$ conformation (lower right half). c vSGLT structure $2 \mathrm{XQ} 2$ in the Ci conformation. d AdiC structure 30B6 in the $\mathrm{CSe}$ conformation
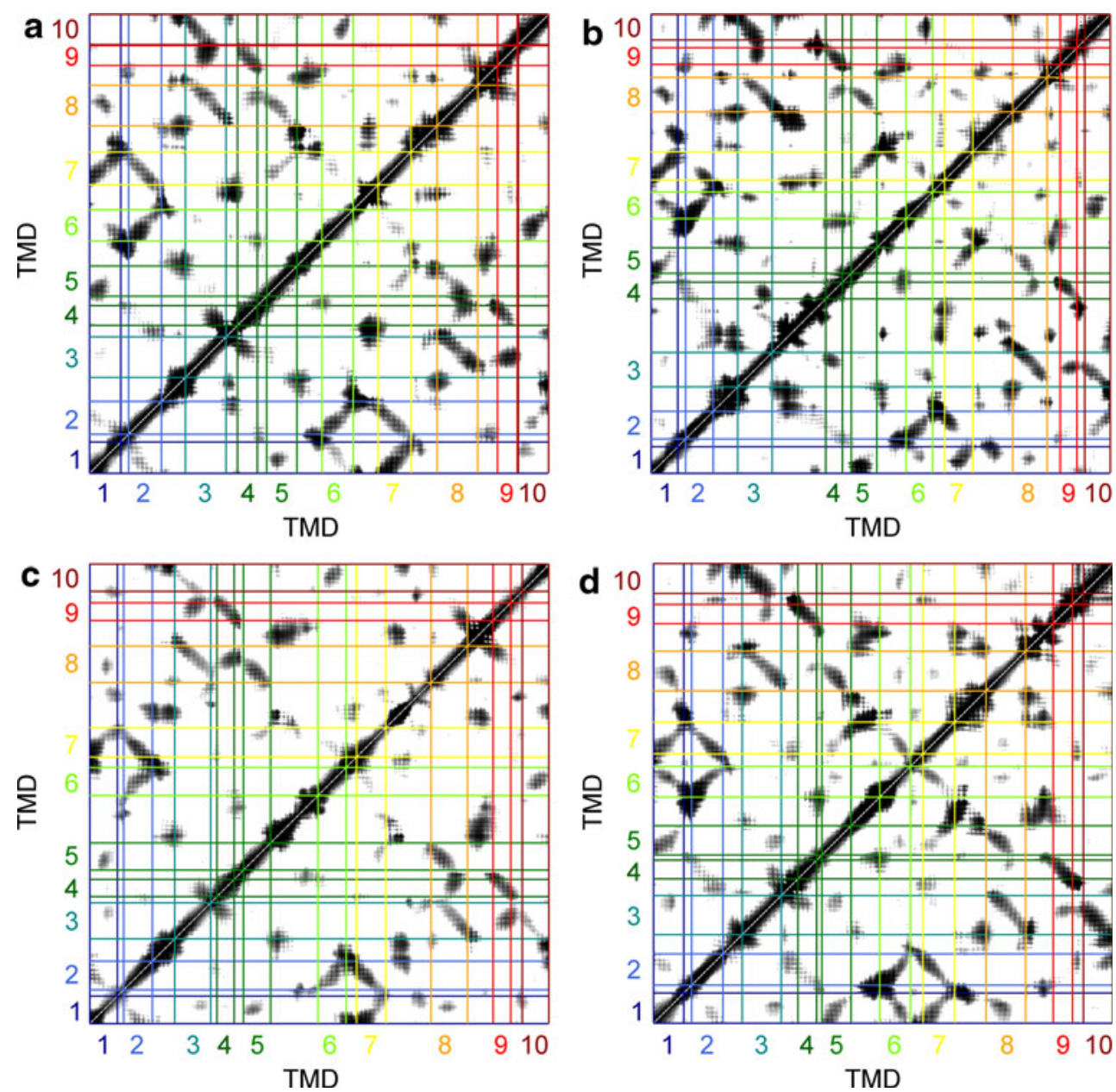

within the hash. In the bundle, TMD 1 invariably correlates with TMD 7 and TMD 2 correlates with TMDs 6 and 7. A weaker correlation is usually seen between TMD 1 and TMD 6. These two TMDs are significantly kinked, so that their common interaction surface is reduced.

In the hash, TMD 3 correlates with TMDs 8 and 9; TMD 4 strongly correlates with TMD 9 and more weakly correlates with TMD 8. Arm I (TMD 5) couples to TMD 1 of the bundle. For outward-open states this coupling is mainly via the respective N-terminal moieties (Fig. 5a, b upper left halves and $\mathrm{d}$ ), whereas for the inward-open states the centers of the two TMDs are more strongly coupled (Fig. 5a, b lower right halves and c). TMD 5 also couples to TMD 7 of the bundle and TMD 8 of the hash.

Likewise arm II (TMD 10) couples to both bundle and hash TMDs. The correlation of TMD 10 with TMD 6 is expected from pseudosymmetry of the inverted repeats and the analogous correlation of TMD 5 with TMD 1. Likewise, the correlation of TMD 10 with TMD 2 is analogous to the one between TMD 5 and TMD 7; however, it is much weaker for arm II than for arm I. Coupling of arm II to the hash TMD 3 is stronger in outward-open structures
(Fig. 5a, b upper left halves and d) than in inward-open structures (Fig. 5a, b lower right halves and c).

Three other features are common to all covariance matrices. First, loop L3-4 exhibits correlated motion with TMDs 3 and 4, as does the pseudosymmetry-related loop L8-9 with TMDs 8 and 9. These couplings may relate to the concerted motion of the hash motif, which is composed of TMDs 3, 4, 8, and 9. Second, according to the covariance matrix the periplasmic loops L3-4 and L6-7 exhibit some kind of correlated motion. Since these loops are distant from each other, this correlation must arise from highly collective modes, such as the modes that correspond to the relative movement of hash and bundle. Third, the only direct coupling between a hash and bundle TMD involves TMDs 1 and 8, and is moderate in outward-open structures and weak or absent in inward-open structures. As pointed out by a reviewer, TMD 1 and 8 form the conserved sodium-binding site $\mathrm{Na} 2$ (Abramson and Wright 2009; Zhao and Noskov 2011). The change in coupling of these two TMDs could thus be related to sodium drawing them together to stabilize the open-out conformation (Zhao and Noskov 2011). Indeed, a new LeuT structure shows 
that a move of the periplasmic moiety of the partially unwound helix TMD 1 is coupled to release of the sodium ion and opening of the intracellular gate (Krishnamurthy and Gouaux 2012). Such motion may then well be related to the relative motion between hash and bundle. We have checked that all these features are also observable in the covariance matrices of the other structures discussed in this work (data not shown).

The preference for couplings within the hash and bundle motifs over couplings between these motifs supports the assumption of relatively rigid, independently moving hash and bundle domains. A similar observation was made with coarse-grained Gō models (Adelman et al. 2011).

Coverage of the structural transitions by a reduced set of ENM modes

ENM can be used to characterize conformational changes of proteins with a small number of distance constraints (Zheng and Brooks 2006). Such an approach would be of interest for characterizing states of transporters, which could not yet be crystallized, by site-directed spin labeling and EPR distance measurements. However, as previous tests of the approach have been performed only for soluble proteins and almost exclusively for interdomain hinge motion, it is not clear whether ENM can also cover the conformational changes of transporters. We shall test this hypothesis in the following.

As demonstrated in Fig. 6 for the example of the $\mathrm{Ce} \rightarrow \mathrm{Ci}$ transition of Mhp1, the tendency for mode coefficients $d_{k}$ to decrease with increasing index $k$ is indeed observed for structural changes in the LeuT fold. In other words, slow modes of the ANM cover a substantial part of the conformational changes. However, some of the coefficients with higher numbers are not small. The first moment of the distribution is as large as 218 . This indicates that only part of the coordinate change can be explained by collective motion of an ANM. Nevertheless, the 50 lowest normal modes cover about $50 \%$ of the coordinate change. Similar tendencies were observed for the other structural transitions discussed in this work (data not shown).

Based on our definition of fractional coverage $f_{1}$ of the conformational change by a small basis of slow normal modes (see Sect. "Methods"), we have tested to what extent structural transitions can be described by a reduced basis of 50 normal modes, corresponding to about $5 \%$ of the total number of modes for the core of transporters with the LeuT fold. This number of modes is a compromise between precision of the description and the effort required for obtaining distance constraints in order to perform such fits with the algorithm described in (Zheng and Brooks 2006).

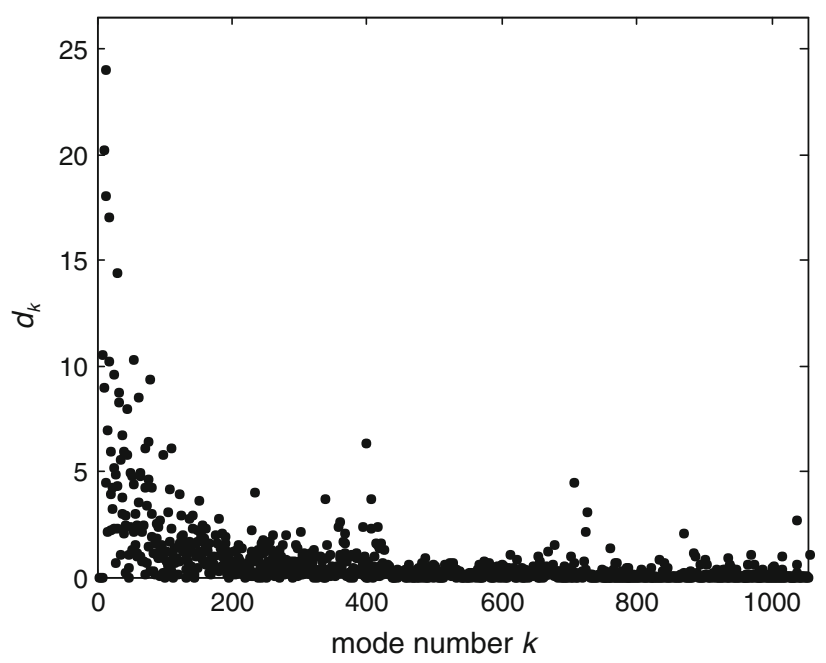

Fig. 6 Dependence of normal mode coefficients $d_{k}$ on mode number $k$ for expressing the coordinate change between Mhp1 structures $2 \mathrm{JLN}$ and $2 \mathrm{X} 79$ as a linear combination of ANM normal modes

The results are compiled in Table 3 for all transitions with significant motion of core TMDs. In most cases, $45-50 \%$ of the structural change can be reproduced with the lowest 50 modes. However, only $26 \%$ of the change is covered for the $\mathrm{Ce}(3 \mathrm{LRB}) \rightarrow \mathrm{CSe}$ (3OB6) transition in AdiC and only $36 \%$ for the CSic (3DH4) $\rightarrow$ Ci (2XQ2) transition in vSGLT. Unsurprisingly, the ANMs fare worse when the structural change is mainly caused by substrate binding, which is dominated by formation of specific interactions that are ignored in the ANMs. Substrate binding to Mhp1 from the outside (2JLN $\rightarrow$ 2JLO) appears to be an exception. We may not exclude that this exception arises from only partial remodeling of the structure for those parts of the electron density that exhibit the strongest differences. Recomputation of the normal modes does not improve coverage for $\Delta_{\exp }<1.5 \AA$, but does so for larger structural changes.

The CSec (2JLO) $\rightarrow$ Ci (2X79) transition of Mhp1 is visualized in Fig. 7a, c based on the two crystal structures and in Fig. 7b, d based on the crystal structure of the starting conformation 2JLO and the ANM coordinate set $\boldsymbol{R}_{\mathrm{f}}$ for the end point. Note that this comparison shows how well the ANM could potentially reproduce the conformational change if driven by a sufficient number of experimental distance constraints. At $49 \%$ coverage of the coordinate change, the gist of the structural transformation is well captured. Apart from moderate errors in direction and amplitude of some of the motion cones, the main deficiency lies in a significant underestimate of the inward motion of L5-6 (blue arrow in Fig. 7b) and a correlated reorientation of TMD 5. Note that the conformational change of L5-6 might indeed be uncoupled from TMD motion. This appears to be feasible since loop conformations differ without an 
Table 3 Coverage of coordinate changes during structural transitions by the 50 lowest normal modes of ANMs

\begin{tabular}{|c|c|c|c|c|c|c|}
\hline Transition & $b(\%)^{\mathrm{a}}$ & $\Delta_{\exp }(\AA)^{\mathrm{b}}$ & $\Delta_{B, 0}(\AA)^{\mathrm{c}}$ & $f_{0}(\%)^{\mathrm{d}}$ & $\Delta_{B, 1}(\AA)^{\mathrm{e}}$ & $f_{1}(\%)^{\mathrm{f}}$ \\
\hline $2 \mathrm{JLN} \rightarrow 2 \mathrm{JLO}$ & 4.8 & 1.23 & 0.63 & 48.9 & 0.66 & 46.6 \\
\hline $2 \mathrm{JLN} \rightarrow 2 \mathrm{X} 79$ & 4.8 & 3.22 & 2.00 & 37.9 & 1.63 & 49.3 \\
\hline $3 \mathrm{~F} 3 \mathrm{~A} \rightarrow 2 \mathrm{~A} 65$ & 4.1 & 1.21 & 0.66 & 45.3 & 0.65 & 46.5 \\
\hline $3 \mathrm{LRB} \rightarrow 3 \mathrm{OB} 6$ & 5.0 & 1.75 & 1.39 & 20.8 & 1.29 & 26.4 \\
\hline $3 \mathrm{OB} 6 \rightarrow 3 \mathrm{~L} 1 \mathrm{~L}$ & 4.8 & 2.32 & 1.39 & 40.0 & 1.28 & 44.7 \\
\hline $3 \mathrm{DH} 4 \rightarrow 2 \mathrm{XQ} 2$ & 4.4 & 1.18 & 0.76 & 35.3 & 0.75 & 36.3 \\
\hline
\end{tabular}

${ }^{a}$ Percentage of all modes that is contributed by the basis of 50 modes

${ }^{b} \mathrm{C}^{\alpha}$ r.m.s.d. between the two structures

${ }^{c} \mathrm{C}^{\alpha}$ r.m.s.d. covered by 50 normal modes of the ANM of the initial structure

${ }^{\mathrm{d}}$ Fractional coverage by 50 normal modes of the ANM of the initial structure

${ }^{\text {e }} \mathrm{C}^{\alpha}$ r.m.s.d. covered by 50 iteratively recomputed normal modes

${ }^{\mathrm{f}}$ Fractional coverage by 50 iteratively recomputed normal modes

Fig. 7 Visualization of the $\mathrm{CSec} \rightarrow \mathrm{Ci}$ transition of Mhp1 from the crystal structures of the starting and end conformation (left) and from the crystal structure of the starting conformation and the ANM fit result $\boldsymbol{R}_{\mathrm{f}}$ for the end conformation (left). a View parallel to the membrane (periplasmic side up) of a coil model of structure 2JLO (CSec conformation) with motion cones pointing to $\mathrm{C}^{\alpha}$ atom locations in structure 2 X79 $(\mathrm{Ci}$ conformation). b View parallel to the membrane of a coil model of structure $2 \mathrm{JLO}$ with motion cones pointing to $\mathrm{C}^{\alpha}$ atom locations in the ANM fit result $\boldsymbol{R}_{\mathrm{f}}$. c View normal to the membrane from the periplasmic side of a coil model of structure 2JLO with motion cones pointing to $\mathrm{C}^{\alpha}$ atom locations in structure $2 \times 79$. d View normal to the membrane of a coil model of structure 2JLO with motion cones pointing to $\mathrm{C}^{\alpha}$ atom locations in the ANM fit result $\boldsymbol{R}_{\mathrm{f}}$
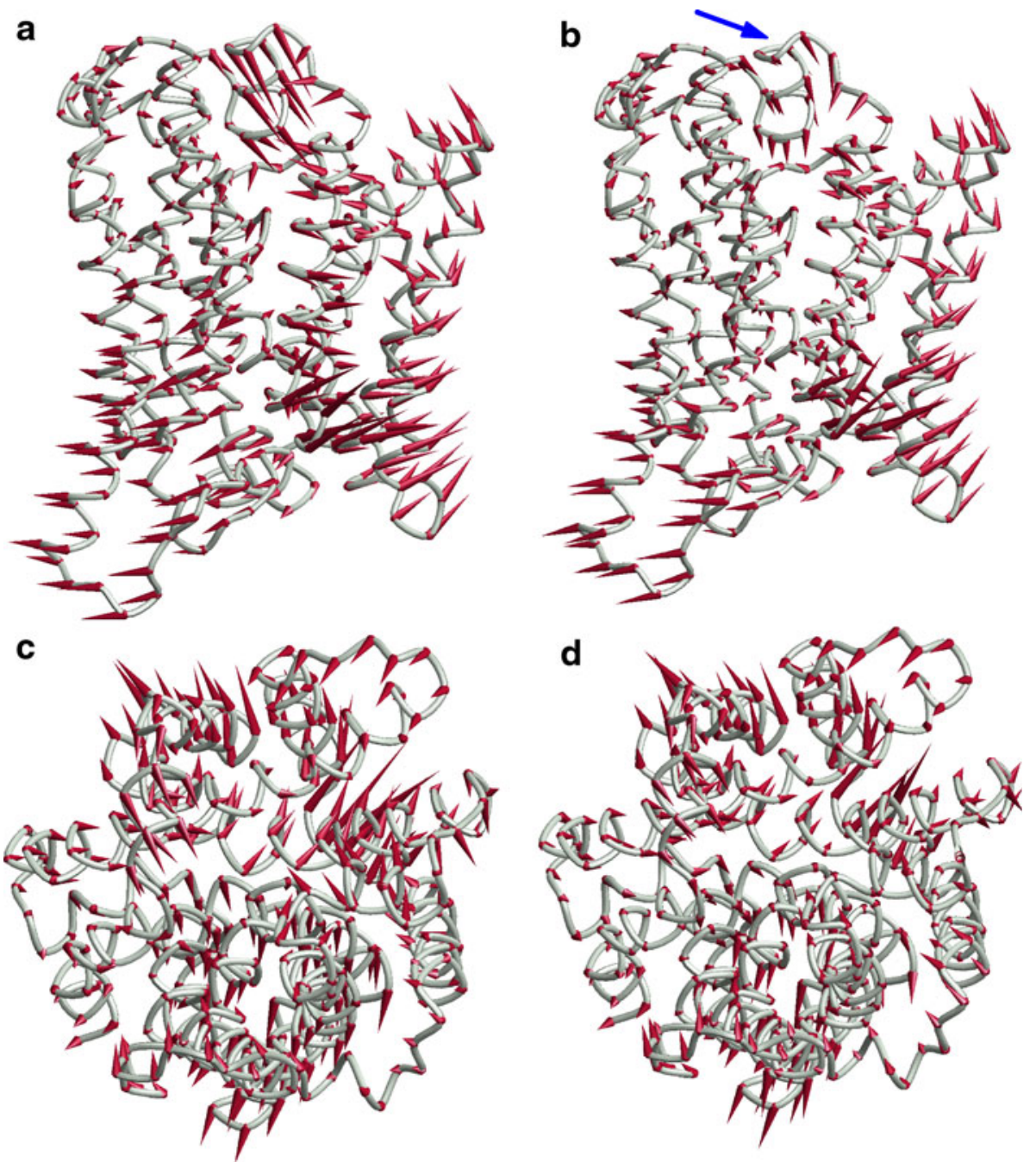

accompanying difference of TMD coordinates in several pairs of crystal structures.

The extent of coverage of the structural transition by the reduced ANM can also be assessed by the characteristic angles. With the ANM model, $\theta_{B, 4}$ decreases by $14.3^{\circ}$, whereas the decrease is $17.1^{\circ}$ for the crystal structure 2 X79. Hence, the ANM covers most of the motion of the hash relative to the bundle. Of the increase in $\phi_{10}$ by 


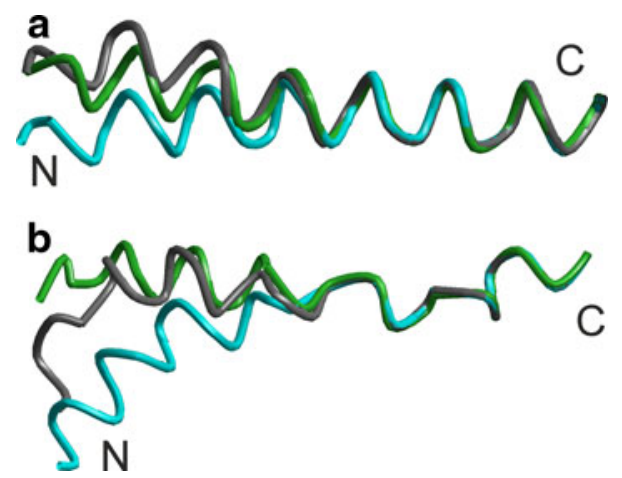

Fig. 8 Coverage of TMD internal conformation changes during the CSe (3OB6) $\rightarrow$ CSec (3L1L) transition of AdiC. Color code Crystal structure 30B6 cyan, crystal structure 3L1L green, ANM fit result $\boldsymbol{R}_{\mathrm{f}}$ grey. a TMD 2. b TMD 6

$17.6^{\circ}$, the ANM covers $10.4^{\circ}$ and of the decrease in $\phi_{5}$ by $24.1^{\circ}$ it covers $11.9^{\circ}$. Again unsurprisingly, the more collective motion of the hash with respect to the bundle is better reproduced than the less collective motion of the arms. Note however that the increase of $18.9^{\circ}$ in $\phi_{10}$ for the $\mathrm{Ce}(2 \mathrm{JLO}) \rightarrow$ CSec (2JLN) transition of Mhp1 is well covered $\left(17^{\circ}\right)$. In this case the structural change is confined to TMD 10 and L9-10, and 50 normal modes are apparently sufficient to reproduce this less complex motion.

We have also tested to which extent internal conformation changes of TMDs are reproduced by ANM fitting with $5 \%$ of the normal modes. The most pronounced changes are observed for the CSe (3OB6) $\rightarrow$ CSec (3L1L) transition of AdiC, in particular for TMDs 2 and 6. As can be seen in Fig. 8 again the gist of these changes is captured by ANM fitting with a reduced basis. For TMD 2 the fit slightly overestimates the change. The $\mathrm{N}$ terminal end of TMD 6 unwinds in the ANM fit, probably owing to problems in covering the conformational change of L5-6.

All these findings combined suggest that the lowest $5 \%$ of the normal modes of ANMs can provide a reasonable, coarse description of large-scale structural changes in secondary transporters with the LeuT fold. This approach has an inherent bias to underestimate the amplitude of structural changes. Interpretation of the results at the residue level should be avoided.

Large-scale structural changes during secondary active transport

Coarse-grained analysis of the structural changes in terms of TMD axes movements (angles $\theta_{B, 4}, \phi_{5}$, and $\phi_{10}$ ) and analysis of the per-residue covariance matrix of ANM strongly suggest that the relative motion of the bundle of TMDs 1,2, 6, and 7 with respect to the hash motif of TMDs $3,4,8$, and 9 is a general feature of transporters with the LeuT fold. In particular, angle $\theta_{B, 4}$ between the mean bundle axis and the mean axis of the invariably straight TMD 4 in the hash motif is strongly correlated with the conformational states of the proteins. This angle decreases by about $20^{\circ}$ during the transition from outward-open to inward-open states. The correlation of changes in angles $\phi_{5}$ and $\phi_{10}$ with the conformational states indicates that arms I (TMD 5) and II (TMD 10) are generally involved in occlusion of the cytoplasmic and periplasmic pathway to the central binding site, respectively. These findings support and extend the rocking-bundle model for the major structural transition in these proteins, which was originally suggested in (Forrest et al. 2008) and extended in (Shimamura et al. 2010).

We also find that the bundle and hash do not strictly move as rigid bodies. In almost all structural changes at least one TMD slightly reorients with respect to the other TMDs in either hash or bundle, and quite often, TMDs slightly flex to occlude or open pathways to the central binding site, in particular, TMDs $1,2,8$, and 10 . These findings qualitatively agree with observations made on unbiased MD simulation trajectories for a homology model of the human serotonin transporter SERT (Kolds $\varnothing$ et al. 2011). They are also in line with the ANM covariance matrices, which indicate differences in coupling strength between different pairs of TMDs within the hash and bundle motif. In the amino acid antiporter AdiC, TMD 6 undergoes a large-scale change of its internal conformation to occlude the periplasmic pathway.

\section{Conclusion}

Superposition of the ten-helix cores of secondary transporter structures in the LeuT fold reveals that relative arrangement of the TMDs is dominated by the functional state, i.e., outward- or inward-open conformation and presence or absence of occlusion of the cytoplasmic and periplasmic path to the central binding site rather than by peculiarities of the individual proteins. Three angles that characterize this relative arrangement correlate well with the functional states. This correlation and analysis of transitions between crystal structures of the same protein for Mhp1, LeuT, AdiC, and vSGLT support the rocking bundle model, which stipulates that the major conformational change in the outward-open to inward-open transition is the relative motion of the bundle of core TMDs 1,2 , 6 , and 7 with respect to the hash motif consisting of TMDs $3,4,8$, and 9. Furthermore, the arm TMDs 5 and 10 appear to play an important role in occlusion of the cytoplasmic and periplasmic pathways to the central binding site. Such occlusion is further aided by slight reorientation of TMDs within the hash and bundle, and by slight internal 
conformation changes of kinked TMDs and moderate internal conformation changes of partially unwound TMDs.

This picture of relative TMD motion is in qualitative agreement with the coupling between TMDs suggested by the per-residue covariance matrix of anisotropic elastic network models, which exhibits similar features for all known structures in the LeuT fold. The observed relative motions of the TMDs and, to some extent, of the intervening loops can be qualitatively reproduced by the $5 \%$ lowest frequency normal modes of the network models, although these modes cover slightly less than $50 \%$ of the coordinate change. The slow normal modes provide only a poor description for conformational changes that are dominated by substrate binding.

These results set the stage for testing hypotheses on structural transitions by EPR distance measurements between spin labels and for modeling the changes by constraint-based fitting of anisotropic elastic network models.

Acknowledgments The author acknowledges helpful discussions with Daniel Hilger and Heinrich Jung, important comments from two anonymous reviewers, and financing from SNF project 20002221E_ 129360.

Open Access This article is distributed under the terms of the Creative Commons Attribution License which permits any use, distribution, and reproduction in any medium, provided the original author(s) and the source are credited.

\section{References}

Abramson J, Wright EM (2009) Structure and function of $\mathrm{Na}(+)$ symporters with inverted repeats. Curr Opin Struct Biol 19:425-432

Abramson J, Smirnova I, Kasho V, Verner G, Kaback HR, Iwata S (2003) Structure and mechanism of the lactose permease of Escherichia coli. Science 301:610-615

Adelman JL, Dale AL, Zwier MC, Bhatt D, Chong LT, Zuckerman DM, Grabe M (2011) Simulations of the alternating access mechanism of the sodium symporter Mhp1. Biophys J 101:2399-2407

Amadei A, Linssen AB, Berendsen HJ (1993) Essential dynamics of proteins. Proteins 17:412-425

Bahar I, Lezon TR, Bakan A, Shrivastava IH (2010) Normal mode analysis of biomolecular structures: functional mechanisms of membrane proteins. Chem Rev 110:1463-1497

Claxton DP, Quick M, Shi L, de Carvalho FD, Weinstein H, Javitch JA, Mchaourab HS (2010) Ion/substrate-dependent conformational dynamics of a bacterial homolog of neurotransmitter:sodium symporters. Nat Struct Mol Biol 17:822-829

Cross TA, Sharma M, Yi M, Zhou HX (2011) Influence of solubilizing environments on membrane protein structures. Trends Biochem Sci 36:117-125

Faham S, Watanabe A, Besserer GM, Cascio D, Specht A, Hirayama BA, Wright EM, Abramson J (2008) The crystal structure of a sodium galactose transporter reveals mechanistic insights into $\mathrm{Na}+$ /sugar symport. Science 321:810-814
Fang Y, Jayaram H, Shane T, Kolmakova-Partensky L, Wu F, Williams C, Xiong Y, Miller C (2009) Structure of a prokaryotic virtual proton pump at 3.2 A resolution. Nature 460:1040-1043

Forrest LR, Rudnick G (2009) The rocking bundle: a mechanism for ion-coupled solute flux by symmetrical transporters. Physiology 24:377-386

Forrest LR, Zhang YW, Jacobs MT, Gesmonde J, Xie L, Honig BH, Rudnick G (2008) Mechanism for alternating access in neurotransmitter transporters. Proc Natl Acad Sci USA 105:1033810343

Forrest LR, Kramer R, Ziegler C (2011) The structural basis of secondary active transport mechanisms. Biochim Biophys Acta 1807:167-188

Gao X, Zhou L, Jiao X, Lu F, Yan C, Zeng X, Wang J, Shi Y (2010) Mechanism of substrate recognition and transport by an amino acid antiporter. Nature 463:828-832

Henzler-Wildman K, Kern D (2007) Dynamic personalities of proteins. Nature 450:964-972

Hilger D, Jung H, Padan E, Wegener C, Vogel KP, Steinhoff HJ, Jeschke G (2005) Assessing oligomerization of membrane proteins by four-pulse DEER: pH-dependent dimerization of NhaA $\mathrm{Na}^{+} / \mathrm{H}^{+}$antiporter of E. coli. Biophys J 89:1328-1338

Hilger D, Polyhach Y, Padan E, Jung H, Jeschke G (2007) Highresolution structure of a $\mathrm{Na}^{+} / \mathrm{H}^{+}$antiporter dimer obtained by pulsed electron paramagnetic resonance distance measurements. Biophys J 93:3675-3683

Hilger D, Polyhach Y, Jung H, Jeschke G (2009) Backbone structure of transmembrane domain IX of the $\mathrm{Na}^{+}$/proline transporter PutP of Escherichia coli. Biophys J 96:217-225

Hinsen K, Petrescu AJ, Dellerue S, Bellissent-Funel MC, Kneller GR (2000) Harmonicity in slow protein dynamics. Chem Phys 261:25-37

Huang Y, Lemieux MJ, Song J, Auer M, Wang DN (2003) Structure and mechanism of the glycerol-3-phosphate transporter from Escherichia coli. Science 301:616-620

Jardetzky O (1966) Simple allosteric model for membrane pumps. Nature 211:969-970

Jeschke G, Polyhach Y (2007) Distance measurements on spinlabelled biomacromolecules by pulsed electron paramagnetic resonance. Phys Chem Chem Phys 9:1895-1910

Jeschke G, Wegener C, Nietschke M, Jung H, Steinhoff HJ (2004) Interresidual distance determination by four-pulse double electron-electron resonance in an integral membrane protein: the $\mathrm{Na}^{+}$/proline transporter PutP of Escherichia coli. Biophys J $86: 2551-2557$

Klingenberg M (2006) Transport catalysis. Biochim Biophys Acta 1757:1229-1236

Kolds $\varnothing$ H, Noer P, Grouleff J, Autzen HE, Sinning S, Schiøtt B (2011) Unbiased simulations reveal the inward-facing conformation of the human serotonin transporter and $\mathrm{Na}(+)$ ion release. PLoS Comput Biol 7:e1002246

Kowalczyk L, Ratera M, Paladino A, Bartoccioni P, ErrastiMurugarren E, Valencia E, Portella G, Bial S, Zorzano A, Fita I, Orozco M, Carpena X, Vazquez-Ibar JL, Palacin M (2011) Molecular basis of substrate-induced permeation by an amino acid antiporter. Proc Natl Acad Sci USA 108:3935-3940

Krishnamurthy H, Gouaux E (2012) X-ray structures of LeuT in substrate-free outward-open and apo inward-open states. Nature 481:469-474

Li J, Tajkhorshid E (2009) Ion-releasing state of a secondary membrane transporter. Biophys J 97:L29-L31

Mchaourab HS, Steed PR, Kazmier K (2011) Toward the fourth dimension of membrane protein structure: insight into dynamics from spin-labeling EPR spectroscopy. Structure 19:1549-1561

Quick M, Winther AM, Shi L, Nissen P, Weinstein H, Javitch JA (2009) Binding of an octylglucoside detergent molecule in the 
second substrate (S2) site of LeuT establishes an inhibitor-bound conformation. Proc Natl Acad Sci USA 106:5563-5568

Ressl S, Terwisscha van Scheltinga AC, Vonrhein C, Ott V, Ziegler C (2009) Molecular basis of transport and regulation in the $\mathrm{Na}(+) /$ betaine symporter BetP. Nature 458:47-52

Rudnick G (2011) Cytoplasmic permeation pathway of neurotransmitter transporters. Biochemistry 50:7462-7475

Schulze S, Koster S, Geldmacher U, Terwisscha van Scheltinga AC, Kuhlbrandt W (2010) Structural basis of $\mathrm{Na}(+)$-independent and cooperative substrate/product antiport in CaiT. Nature 467:233236

Shaffer PL, Goehring A, Shankaranarayanan A, Gouaux E (2009) Structure and mechanism of a $\mathrm{Na}^{+}$-independent amino acid transporter. Science 325:1010-1014

Shan J, Javitch JA, Shi L, Weinstein H (2011) The substrate-driven transition to an inward-facing conformation in the functional mechanism of the dopamine transporter. PLoS ONE 6:e16350

Shi L, Weinstein H (2010) Conformational rearrangements to the intracellular open states of the LeuT and ApcT transporters are modulated by common mechanisms. Biophys J 99:L103-L105

Shimamura T, Weyand S, Beckstein O, Rutherford NG, Hadden JM, Sharples D, Sansom MS, Iwata S, Henderson PJ, Cameron AD (2010) Molecular basis of alternating access membrane transport by the sodium-hydantoin transporter Mhp1. Science 328:470473

Singh SK, Piscitelli CL, Yamashita A, Gouaux E (2008) A competitive inhibitor traps LeuT in an open-to-out conformation. Science 322:1655-1661

Smirnova I, Kasho V, Choe JY, Altenbach C, Hubbell WL, Kaback HR (2007) Sugar binding induces an outward facing conformation of LacY. Proc Natl Acad Sci USA 104:16504-16509

Tang L, Bai L, Wang WH, Jiang T (2010) Crystal structure of the carnitine transporter and insights into the antiport mechanism. Nat Struct Mol Biol 17:492-496
Wang H, Elferich J, Gouaux E (2012) Structures of leuT in bicelles define conformation and substrate binding in a membrane-like context. Nat Struct Mol Biol 19:212-219

Watanabe A, Choe S, Chaptal V, Rosenberg JM, Wright EM, Grabe M, Abramson J (2010) The mechanism of sodium and substrate release from the binding pocket of vSGLT. Nature 468:988-991

Weyand S, Shimamura T, Yajima S, Suzuki S, Mirza O, Krusong K, Carpenter EP, Rutherford NG, Hadden JM, O'Reilly J, Ma P, Saidijam M, Patching SG, Hope RJ, Norbertczak HT, Roach PC, Iwata S, Henderson PJ, Cameron AD (2008) Structure and molecular mechanism of a nucleobase-cation-symport-1 family transporter. Science 322:709-713

Yamashita A, Singh SK, Kawate T, Jin Y, Gouaux E (2005) Crystal structure of a bacterial homologue of $\mathrm{Na}^{+} / \mathrm{Cl}-$ dependent neurotransmitter transporters. Nature 437:215-223

Yang L, Song G, Jernigan RL (2007) How well can we understand large-scale protein motions using normal modes of elastic network models? Biophys J 93:920-929

Zhao C, Noskov SY (2011) The role of local hydration and hydrogenbonding dynamics in ion and solute release from ion-coupled secondary transporters. Biochemistry 50:1848-1856

Zhao Y, Terry D, Shi L, Weinstein H, Blanchard SC, Javitch JA (2010) Single-molecule dynamics of gating in a neurotransmitter transporter homologue. Nature 465:188-193

Zhao Y, Terry DS, Shi L, Quick M, Weinstein H, Blanchard SC, Javitch JA (2011) Substrate-modulated gating dynamics in a $\mathrm{Na}^{+}$-coupled neurotransmitter transporter homologue. Nature 474:109-113

Zheng W, Brooks BR (2006) Modeling protein conformational changes by iterative fitting of distance constraints using reoriented normal modes. Biophys J 90:4327-4336

Zhou Z, Zhen J, Karpowich NK, Goetz RM, Law CJ, Reith ME, Wang DN (2007) LeuT-desipramine structure reveals how antidepressants block neurotransmitter reuptake. Science 317:1390-1393 